\title{
Digital Subtraction Angiography: A Review of Cardiac Applications
}

\author{
G.B. John Mancini and Charles B. Higgins
}

$I^{N}$ MAGING OF the chambers of the heart, pulmonary circulation, and great vessels by intravenous (IV) contrast injection was clinically implemented as early as $1939 .{ }^{1}$ It was not until the achievement of relatively recent advances in digital electronics, image intensification, and television technology that a major resurgence of interest in this technique occurred. These technological advances in conjunction with the pioneering work from the Universities of Kiel, Federal Republic of Germany; Arizona at Tucson; Wisconsin at Madison; as well as the Mayo Clinic in Rochester, Minn, provided the groundwork for exciting applications of digital subtraction angiography in the assessment of cardiovascular dynamics. ${ }^{2-10}$ These results initiated widespread clinical evaluation of the use of this imaging modality in cardiac diagnosis that was facilitated by commercially available digital angiography systems which provided rudimentary image manipulation capabilities. The purpose of this report is to summarize the recent applications of digital subtraction angiography in the quantitative assessment of global and regional left ventricular function, coronary anatomy, and coronary flow dynamics. Earlier work with videodensitometry or off-line digital analysis is also included when relevant to current applications of digital angiography. Practical issues regarding technical prerequisites, clinical techniques, contrast material, and image manipulation are also discussed as they relate to cardiac investigations.

\section{TECHNICAL CONSIDERATIONS}

A discussion of the physics of rocntgen digital imaging and the different digital systems has been provided by several recent publications. ${ }^{11-16}$ It is important to appreciate that developmental advances in hardware and software continue to occur in this field and that requirements of the digital systems used for evaluating cardiac function and coronary arteries are different from those considered optimal for evaluating vascular morphology in other beds. ${ }^{17-18}$ Compared to coronary or peripheral vessels, for example, the ventricles are large and relatively contrast-laden after peripheral or direct contrast injection, so that a matrix size of $256 \times 256$ or $512 \times 512$, in conjunction with a nine-inch image intensifier, generally provide adequate spatial resolution for analysis of ventricular function, although the spatial resolution is decidedly less than afforded by standard cineangiography. The framing rate, however, must be sufficiently rapid to accurately depict regional ventricular function. In contrast, temporal resolution is of less importance in vascular imaging, but the demands on spatial resolution are much greater and generally mandate matrix sizes of $512 \times 512$ or more. Temporal resolution in this application may also be important in selected investigations (eg, blood flow assessment). The option to provide ECG-gated image acquisitions is also of benefit in certain applications to be described.

Several recent articles from the University of Arizona highlight and amplify these different aspects of digital systems designed for general purpose angiography compared to cardiac applications. ${ }^{17,18}$ These authors also stress the need for systems that can be easily improved to take advantage of innovations in archival, television, and video technology.

\section{CONTRAST MATERIAL}

Although conventional contrast agents are relatively safe, a substantial proportion of patients subjected to them may suffer from systemic, vascular, renal, and cardiac side effects. ${ }^{19-21}$ These agents contribute significantly to the morbidity and mortality involved in angiography, including coronary angiography and left ventriculography. ${ }^{22-33}$ Contrast effects cannot be ignored in the implementation of IV

From the Division of Cardiology, Department of Internal Medicine, University of Michigan Medical School, Ann Arbor, Mich, and the Department of Radiology, University of California, San Francisco School of Medicine, San Francisco

Address reprint requests to G.B. John Mancini, MD, Cardiology Section, Veterans Administration Medical C'enter, 2215 Fuller Rd, Ann Arbor, MI 48105.

(c) 1985 by Grune \& Stratton, Inc.

0033-0620/85/2802-0002\$05.00/0 
digital subtraction angiography because each image run typically requires the administration of between 20 and $60 \mathrm{~mL}$ of contrast material. ${ }^{34,35}$

Figure 1 demonstrates the changes in right heart hemodynamics determined in ten patients who were each injected with $45 \mathrm{~mL}$ of ionic contrast material (diatrizoate sodium, diatrizoate meglumine, Renografin 76 [ER Squibb, Princeton, $\mathrm{NJ}]$ ) into the inferior vena cava or the left ventricle. Although intraventricular injection caused a significantly greater elevation in mean right atrial pressure and mean pulmonary artery pressure. it is apparent that these differences were actually quite small and not more than 1 to $3 \mathrm{~mm} \mathrm{Hg}$. Moreover, pulmonary wedge pressure was elevated to a similar degree after both injections. Figure 2 shows similar findings in the left heart hemodynamics, ie, despite minor, transient differences in heart rate and cardiac output, there were identical degrees of systemic hypotension and elevation of the left ventricular enddiastolic pressure. This study underscores the

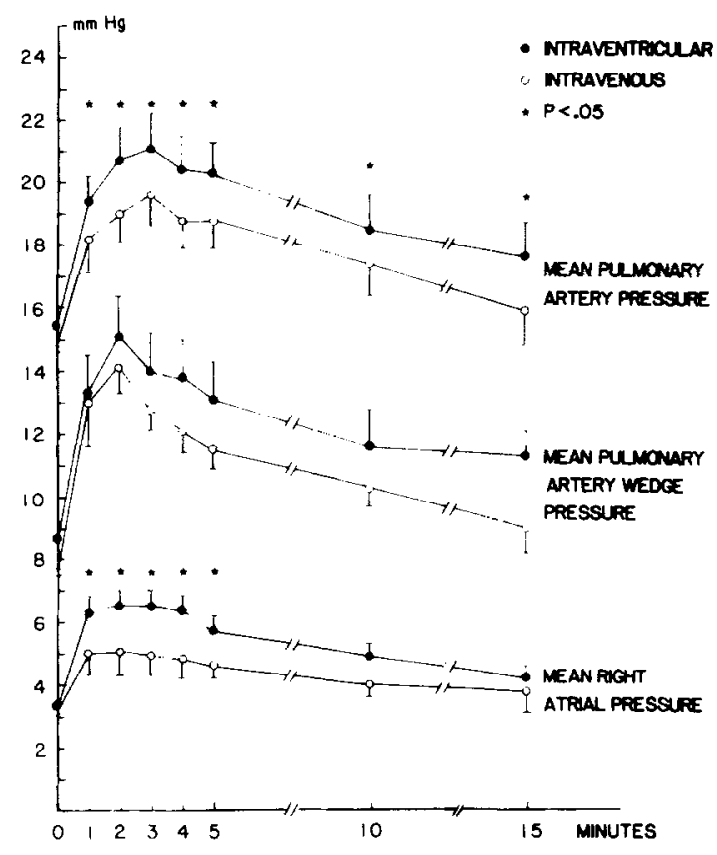

Fig 1. A comparison of changes in right heart hemodynamics after IV or intraventricular injection of $45 \mathrm{~mL}$ of ionic contrast material. Although statistically significant differences are noted in the response of the mean pulmonary artery pressure and the mean right atrial pressure, the differences are actually of small magnitude. (Reprinted with permission. ${ }^{35}$ )

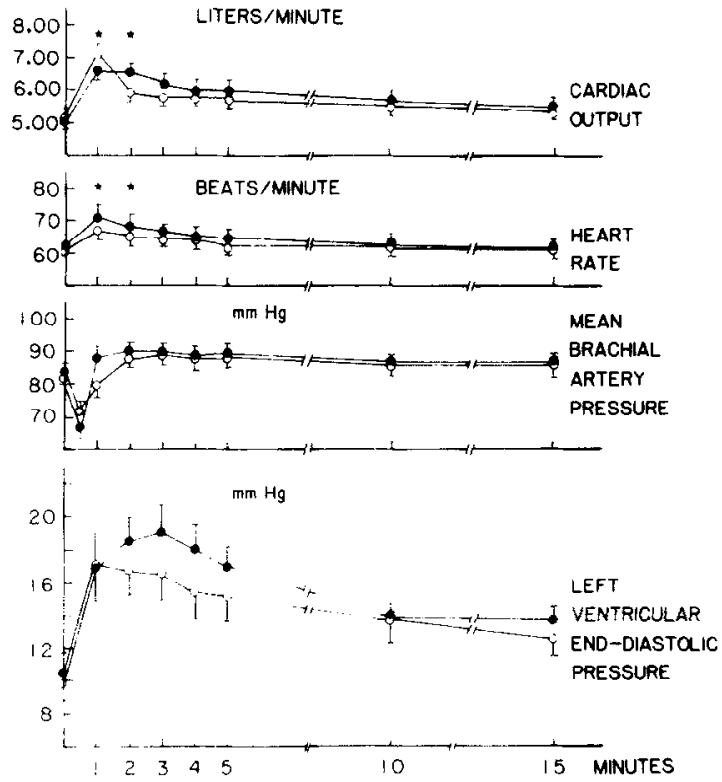

Fig 2. A comparison of changes in left heart hemodynamics. As in Fig 1, the few statistically significant differences between IV and intraventricular ionic contrast injection are of very small magnitude. (Reprinted with permission. ${ }^{36}$ ) e, Intraventricular; O. IV: $\star P<.05$.

fact that although IV angiography obviates the risks of arterial cannulation, the hemodynamic risks attendent to the use of contrast material are not significantly reduced. This drawback can be minimized by using the smallest amount of contrast material compatible with adequate imaging, or by the use of newer agents with fewer side effects. ${ }^{36-41}$

Compared to standard ionic contrast. IV administration of a new nonionic contrast agent (iohexol) has been shown to cause fewer changes in left ventricular pressure, function, and coronary blood flow. ${ }^{42}$ Patient studies have also shown that this newer, nonionic agent has decreased toxicity. ${ }^{43,44}$ Figures 3 and 4 demonstrate the less severe alterations of systemic pressure and the absence of ECG changes induced by iohexol. Favorable comparative effects on myocardial contractility and relaxation have also been demonstrated with this agent. ${ }^{44}$ This diminished toxicity is related to several physical properties of newer nonionic agents, including a substantial lowering of osmolality, negligible amounts of sodium, and a lack of induction of hypocalcemia and hypokalemia. $^{40}$ 


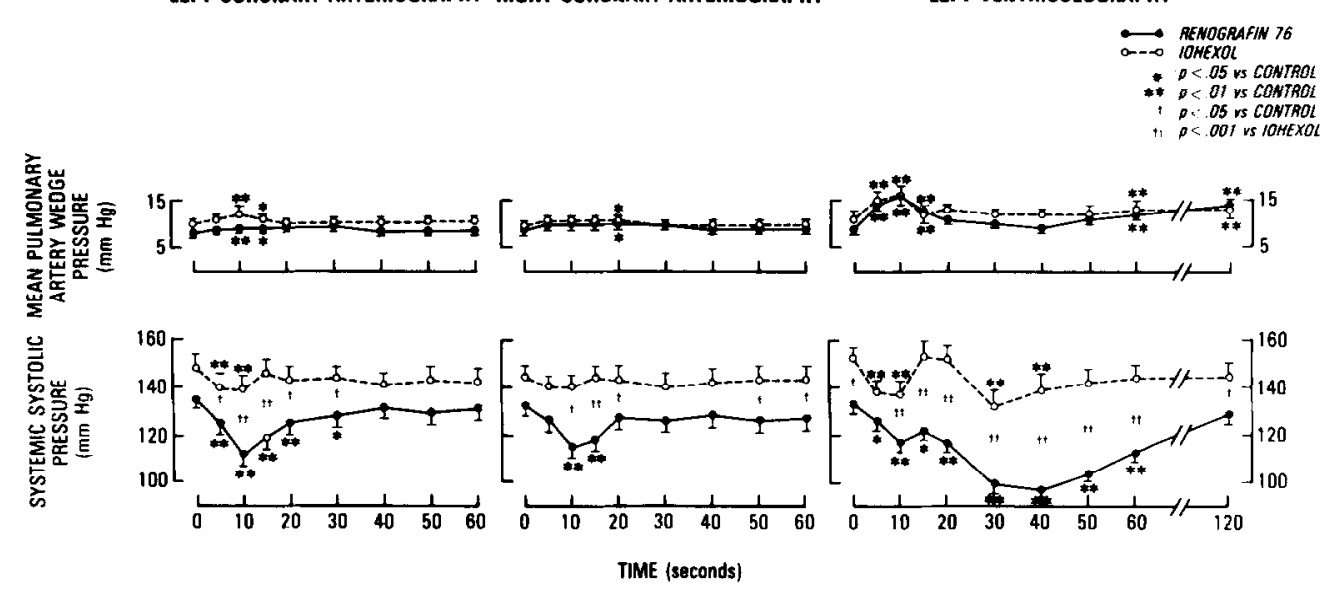

Fig 3. Hemodynamic changes induced by Renografin 76 (Squibb. Princeton, NJ) and iohexol. The nonionic agent iohexol caused fewer changes in systemic blood pressure after coronary arteriography and left ventriculography. (Reprinted with permission. ${ }^{43}$ )

Thus, innovations in the development of contrast materials can lessen the drawbacks of IV digital subtraction angiography related to the use of conventional contrast agents. The use of these newer agents may also have the added advantage of reducing patient discomfort during injection and this might, in turn, minimize patient motion and misregistration artifacts. ${ }^{36}$ This added benefit, however, has not been reported by some investigators. $^{37}$

The intra-arterial applications of digital subtraction angiography virtually obviate hemodynamic toxicities, because very small doses of contrast material can be used selectively to acquire diagnostic images. ${ }^{45-48}$

\section{CLINICAL TECHNIQUES}

Although there was initial enthusiasm for the peripheral IV injection of contrast material to procure images of the systemic circulation, practical experience revealed a small but unacceptable incidence of complications. These include contrast extravasation into the arm and an inability to consistently inject adequate volumes of contrast material. ${ }^{49-53}$ Consequently, contrast material is now more frequently administered by
Fig 4. ECG changes induced by contrast agents. Compared to Renografin 76 (Squibb. Princeton, NJ). iohexol produced no changes in the electrocardiogram. (Reprinted with permission. ${ }^{43}$

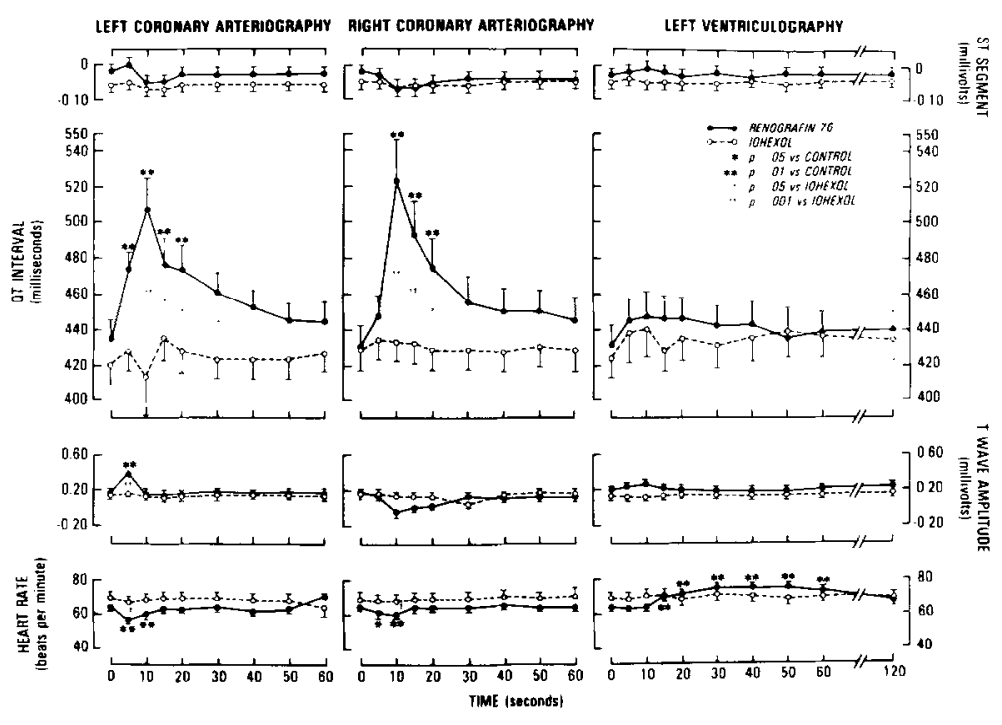


means of a central IV injection into the vena cava or the right atrium. The catheters generally have multiple side holes, are of $5 \mathrm{~F}$ or $6 \mathrm{~F}$ in size, and are in a "pigtail" configuration to avoid recoil or intramural injection.

Little direct evaluation of the optimal technique of contrast injection for IV cardiac studies is currently available. Theoretically, an IV contrast injection that gives a high peak iodine concentration in a narrow or compact bolus would be considered ideal for purposes of subtraction. Such a situation allows selection of mask and contrast phases that are close together in time, resulting in maximal contrast enhancement while minimizing the possibility of misregistration artifacts.

The peak iodine concentration is directly proportional to the amount of iodine injected, and the minimum amount of iodine required depends on the purpose of the study. Between 12 and $15 \mathrm{~g}$ of iodine per injection is usually needed for adequate visualization, especially of small arteries ${ }^{37.54}$ although other investigators have used smaller amounts. ${ }^{50}$ Kruger et $\mathrm{al}^{6}$ present mathematical arguments showing that higher rates of injection result in less dilution of contrast material as it enters the left-sided circulation. In agreement with this are the clinical findings of Saddekni et $\mathrm{al}^{50}$ who confirmed that high peak arterial opacification can be achieved with higher contrast injection rates. Eskridge et al, ${ }^{51}$ however, failed to note any subjective improvement in image quality when higher rates of injection were used. In addition, within a range of injection of $0.16 \mathrm{~mL} / \mathrm{s} / \mathrm{kg}$ to $0.49 \mathrm{~mL} / \mathrm{s} / \mathrm{kg}$ in dogs, Burbank ${ }^{54}$ showed that peak opacification and bolus width was unaffected by the injection rate. This latter study did demonstrate that the peak iodine concentration achievable with a right-sided injection was directly proportional to the amount of iodine injection (not the concentration) and inversely proportional to central blood volume, whereas the width of the contrast bolus was directly proportional to the central blood volume and inversely proportional to cardiac output. Thus, patients with high central blood volume and low cardiac output would be expected to demonstrate nonideal, low peak and wide width iodine bolus curves. It follows from these relationships that lower toxicity contrast materials at relatively high volumes will produce maximal opacification without an increase in bolus width, because these agents cause less pulmonary vasodilatation and only small increases in central blood volume. Finally, Brennecke et $\mathrm{a}^{55}$ have shown that the characteristics of contrast bolus curves are continually altered as the bolus moves through the circulation. Optimization of signal extraction and subtraction techniques for a particular phase of bolus transfer may be suboptimal for a later phase. Thus, all of these factors must be considered in attempting to maximize the diagnostic yield from digital images obtained by IV contrast injection.

Hetzel et al ${ }^{56}$ have shown that peak arterial indicator concentration is best with central venous injections compared to more distal superior vena cava or peripheral venous injections. More recently, Rubin et al ${ }^{57}$ confirmed this in a study of IV injection of contrast material. This is also in agreement with the findings of Eskridge et al, ${ }^{51}$ but this latter group did not note any significant improvements in image quality between right atrial and superior vena cavae injections.

No direct evaluation of the optimal method of low-dose direct intraventricular digital subtraction angiography is currently available. Studies have proposed using injections of composition ranging from 5 to $10 \mathrm{~mL}$ of undiluted contrast injected within 1 to 2 seconds ${ }^{45.46 .48}$ to $7 \mathrm{~mL}$ of contrast, diluted in $43 \mathrm{~mL}$ of saline and injected at $15 \mathrm{~mL} / \mathrm{min} .{ }^{47}$ As with standard cineventriculography, it is anticipated that with this method of ventriculography the injection rates, volumes. and dilutions will be predicated by patient characteristics such as the chamber size, stroke volume, heart rate, and the presence or absence of wall motion abnormalities.

\section{SUBTRACTION TECHNIQUES}

Riederer and $\mathrm{Kruger}^{58}$ have recently summarized the technical characteristics of several alternate subtraction methods and provide a convenient framework for reference (Table 1). Subtraction can be performed with respect to one of several physical variables including time, energy, and depth parameters. "First-order" techniques are those in which only one physical variable is exploited to produce a final difference image. For example, mask-mode imaging used in conventional digital subtraction angiography is a form 
Table 1. Categories of Subtraction Techniques Applied to Digital Subtraction Angiography (DSA)

\begin{tabular}{|c|c|c|c|}
\hline & \multicolumn{3}{|c|}{ Physical Variable } \\
\hline & Time $(t)$ & Energy $(E)$ & Depth (Z) \\
\hline \multirow[t]{4}{*}{ First-order subtraction } & Conventional DSA & Dual beam energy subtraction & Tomography \\
\hline & Integrated remasking & (K-edge and non-K-edge) & \\
\hline & Matched filtering & & \\
\hline & Recursive filtering & & \\
\hline \multicolumn{4}{|l|}{ Second-order subtraction } \\
\hline Z: & Tomographic DSA & - & - \\
\hline $\mathrm{E}:$ & Hybrid subtraction & $\begin{array}{l}\text { Three-beam K-edge energy } \\
\text { subtraction }\end{array}$ & - \\
\hline
\end{tabular}

Adapted with permission. ${ }^{58}$

of temporal subtraction of frames pre- and postcontrast injection. "Second-order" techniques sequentially utilize two physical variables to effect the resultant image. For example, hybrid subtraction $^{59}$ utilizes the physical variables of both time and energy. Clinical cardiac applications of digital subtraction angiography have been almost exclusively involved with temporal subtraction methods. Of the various forms of temporal subtraction, mask-mode and timeinterval-difference (TID) are most commonly reported.

In mask-mode subtraction the digitized image frames during peak opacification are subtracted on a pixel by pixel basis from single or averaged frames of the same area before the arrival of the contrast media (Figs 5 and 6). Alternatively, the mask may be ECG-gated so that each frame of the pre- and post-contrast phases is aligned with respect to the phase of the cardiac cycle (Fig 7). Provided that there is no patient motion, overlying structures devoid of contrast material are subtracted from the image, improving the visibility of faintly opacified, contrast-laden structures. Contrast enhancement (Fig 8) allows density windowing within an operator-defined range containing the most important image information. This small range of grey levels can then be expanded to take advantage of the full grey scale range, thereby enhancing the contrast of the specific area of interest.

TID differs from mast-mode subtraction in that the sets of frames subtracted from each other all contain contrast media, but each set is separated by only a brief period of time (Fig 9). The result of this type of first-order subtraction is a display of the difference in contrast transit or wall motion that occurred between the frames of each set (Fig 10). As a consequence, all stationary portions of the field of view are eliminated, and the final image provides an indication of the instantaneous changes in motion of opacified structures or movement of a contrast bolus throughout the period of image acquisition. An advantage of this method is that noncyclical patient motion is seen only briefly and the shortlived motion artifact does not necessarily degrade the entire image sequence. In contrast, the time-separation between image and mask frames in mask-mode subtraction is relatively long and, therefore, this technique is more prone to degradation due to motion. Misregistration of frames under these circumstances can sometimes be overcome by remasking or by pixel shifting and rotating programs that are becoming available with image processing computers. Other techniques, such as "rubber sheeting" and respiratory-gating, may also be of use. ${ }^{18,61}$
Fig 5. A schematic representation of mask-mode cardiac imaging. Single or averaged frames prior to contrast injection are used as the mask from which frames during maximal cardiac opacification after contrast injection are subtracted. In the resultant image, non-opacified structures are removed leaving behind the contrast containing heart chambers and vessels.

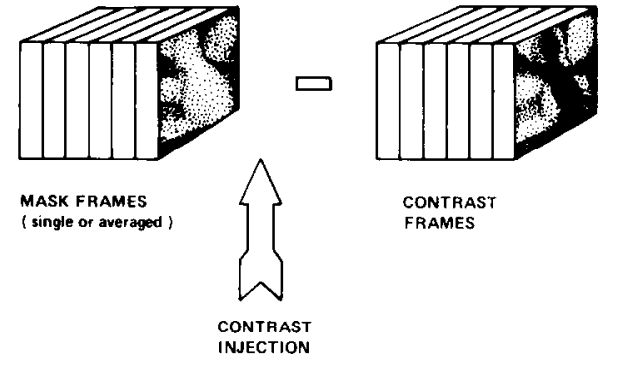

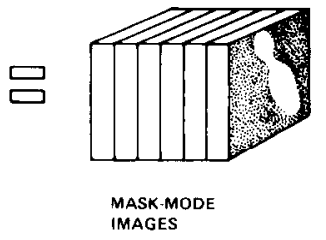




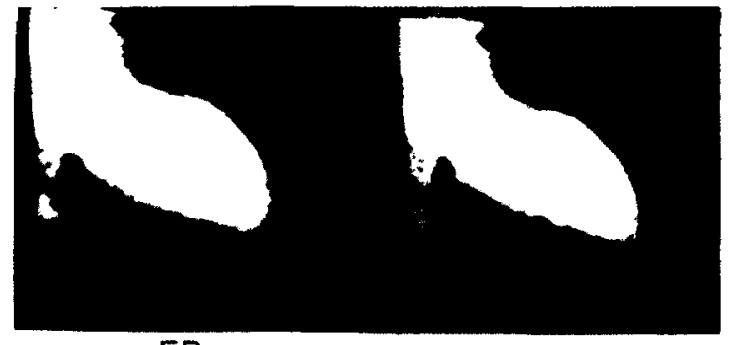

ED

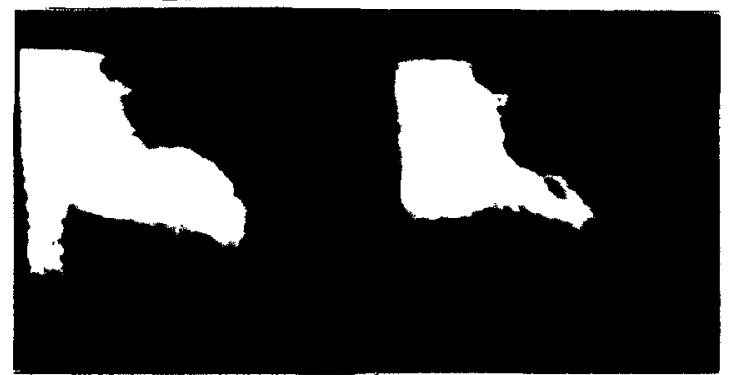

ES

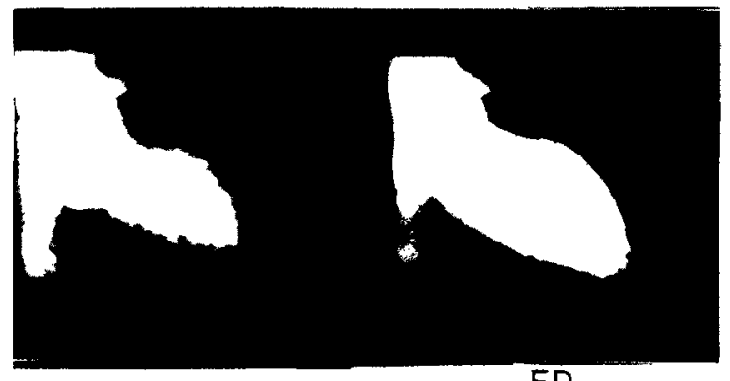

$E D$

MASK MODE

Fig 6. Selected frames from a digital IV ventriculogram showing progression of contraction from end-diastole (ED) to end-systole (ES). (Reprinted with permission. ${ }^{60}$ )

Other types of temporal subtraction techniques include integrated remasking, ${ }^{62,63}$ matched filtering, ${ }^{64-66}$ and recursive filtering ${ }^{67-72}$ (Fig 11). Integrated remasking involves the retrospective analysis of an image run to ascertain which phases are best suited for the mask and the contrast frame sets. Each frame in the mask and contrast phase is equally weighted (average) but of opposite sign. Except for producing the mask, this method is not suited to the contrast phase of cardiac studies because of cyclical motion.

Matched filtering ${ }^{64-66}$ is the process whereby mask and image phases are weighted in proportion to the actual time concentration characteristics of the contrast bolus curve. This temporal integration can be used to either decrease patient exposure or contrast dose while still producing images that are comparable to conventional digital images. Alternatively, at equal roentgen exposure, this technique can be used to increase signal-to-noise relative to conventional temporal subtraction. The extensive temporal integration. however, causes blurring of mobile structures. This will mandate potential modification of the technique, such as ECG-gating, for cardiac applications.

Recursive filtering ${ }^{67-72}$ provides a contrast image which is a combination of the most current image, as well as prior images that are weighted in inverse proportion to the time lag between acquired frames. The mask image is similarly obtained so that the final image is conceptually analogous to a TID image except for the integration and differential weighting of frames. Because the contrast and mask images are constantly updated, this technique has some inherent resistance to image degradation due to periodic motion. Breathing, swallowing, and even panning, do not degrade the entire image sequence. ${ }^{69}$ Cardiac applications of this technique have not been extensive, ${ }^{71-73}$ but are promising (Fig 12).

Hybrid subtraction, recently proposed by Bro$d y,{ }^{59}$ is a second-order subtraction method in

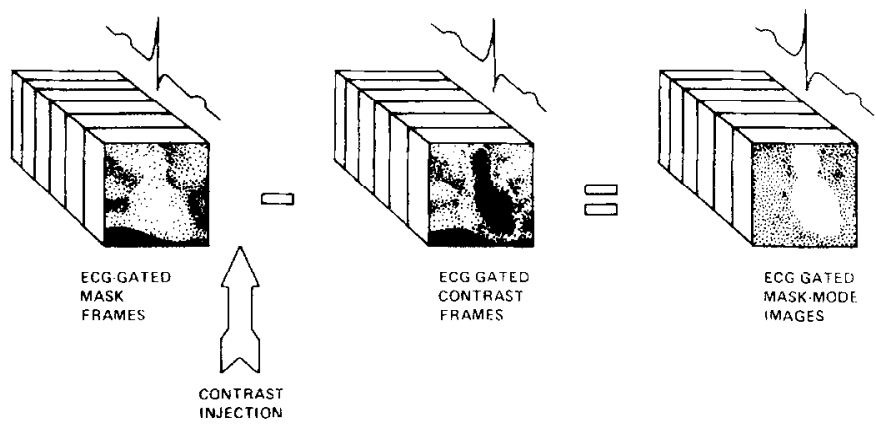

Fig 7. A schematic representation of ECGgated mask mode subtraction. Pre- and postcontrast injection frames are acquired along with an FCG reference signal. The subtractions are performed on image pairs obtained at the same phase of the cardiac cycle. 
Fig 8. A schematic representation of the concept of contrast enhancement. Of the total range of grey levels in the original subtracted image, only a narrow range spans the levels seen in the region of interest of. for example. the left ventricle. This window is selected by the operator and then expanded to fill the entire grey scale, enhancing the contrast.

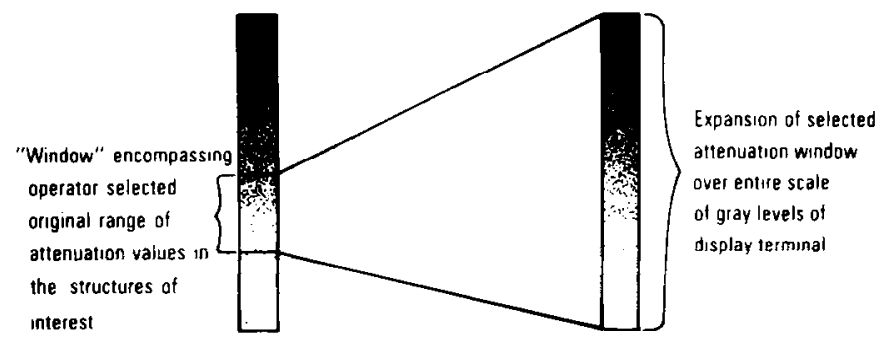

which pairs of high and low energy exposure images are obtained with each alternate frame, separated by a few milliseconds of each other (Fig 13). The image pairs are weighted and combined so that soft tissue is cancelled from the image. This is useful in removing soft tissue misregistration artifacts. Provided that the patient remains stationary so that skeletal structures are properly registered, the images can also be processed by a temporal mask mode subtraction method to effectively cancel overlying bone images. Unfortunately, this method entails greater radiation exposure compared to conventional digital techniques, and subtraction of high and low energy frames post-contrast injection mandates that some of the iodine signal is lost. Furthermore, the second-order subtraction increases image noise. ${ }^{59,74-77}$ Various temporal filtration and integration schemes have been proposed to compensate for these problems. ${ }^{66,77}$ Cardiac applications of hybrid subtraction have been few. Guthaner et $\mathrm{al}^{74}$ report that five out of eight IV examinations for assessment of coronary artery bypass graft patency were improved by

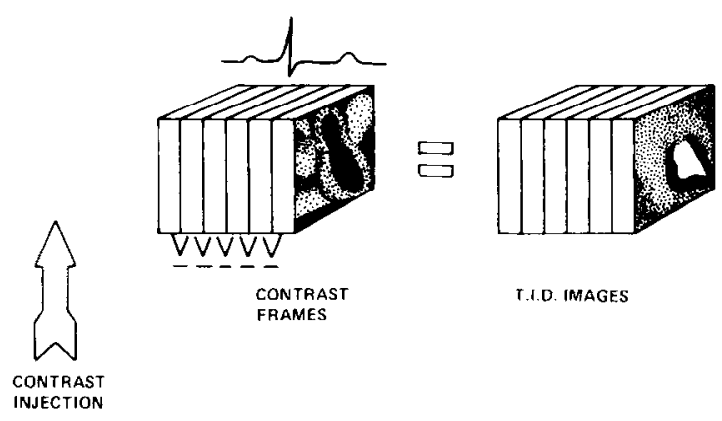

Fig 9. A schematic representation of TID imaging. The image set consists of frames acquired post-contrast injection. Serial pairwise subtractions of consecutive frames are illustrated here. Stationary areas of the images are effectively subtracted leaving behind an indication of the amount of movement that has occurred between the consecutive image pairs. TID processing can be performed on sets of mask-mode subtracted images. the use of hybrid subtraction compared to conventional temporal subtraction (Fig 14).

\section{ASSESSMENT OF LEFT VENTRICULAR FUNCTION}

\section{Experimental Studies}

Several descriptive studies showed the feasibility of assessing left ventricular dynamics in experimental animals. ${ }^{5,15,78-80}$

Bursch et $a^{81}$ used videodensitometry to measure cardiac output in pigs from direct left ventriculograms. Cardiac output correlated well with simultaneously performed indocyanine green dye-dilution methods over a range of 1.5 to $5.0 \mathrm{~L} / \mathrm{min}$. Carey et al ${ }^{82}$ measured heart rate and stroke volumes from digital IV ventriculograms in dogs to calculate cardiac output. These measurements were compared to thermodilution cardiac outputs and found to be highly correlated $(r=.89$, slope $=1.05$, intercept $=0.04)$.

Left ventricular volume measurements from IV ventriculograms in dogs were analyzed by Slutsky et $\mathrm{a} 1^{83}$ and compared to thermodilution and sonomicrometer measurements. Because the same beat was analyzed before and after maskmode subtraction, the effects of this image processing on quantitation of chamber dynamics could be ascertained. The results showed that although measurements from both images correlated quite well, volumes from the mask-mode images consistently underestimated volumes from nonprocessed images by approximately $12 \%$. This study also demonstrated a high correlation $(r=.91)$ between left ventricular enddiastolic volumes determined by area-length analyses and volumes calculated from long axis and minor axis measurements provided by the implanted sonomicrometer crystals.

Radtke et a ${ }^{84,85}$ used direct contrast ventriculography in pigs to assess not only the endocardial contour of the ventricle but also the epicar- 

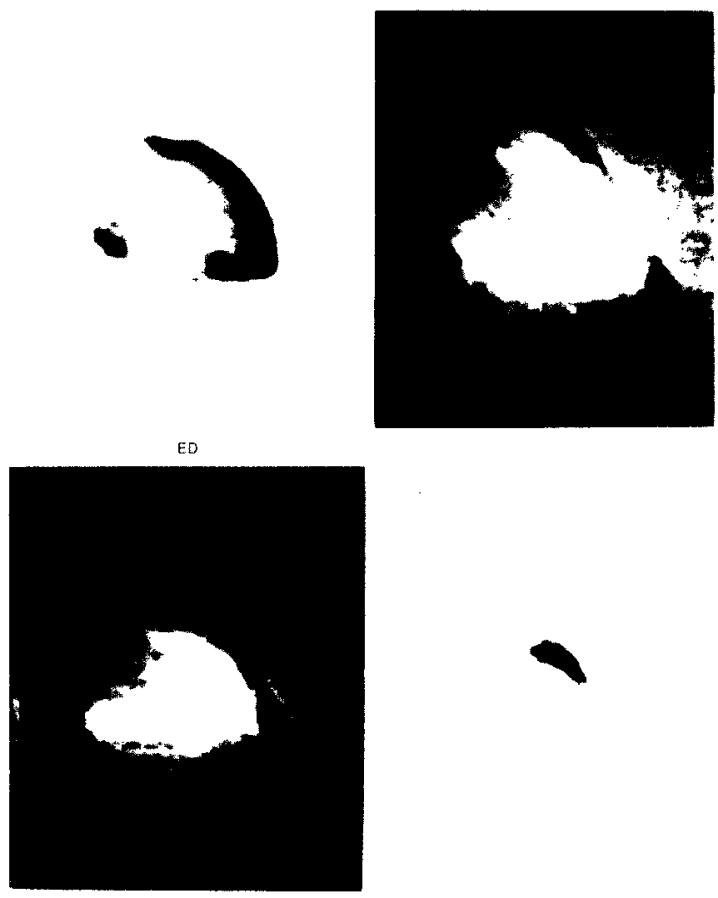

ES
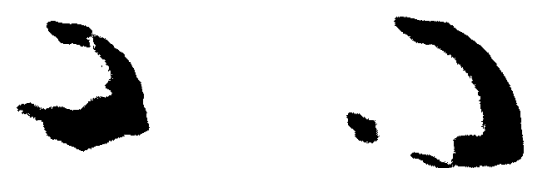

Fig 10. Selected frames from a digital IV ventriculogram processed by the TID method. White rims represent net inward motion, whereas black rims represent net outward motion. The end-systolic frame (ES) shows a crescent of black in the anterolateral region of the ventricle which signifies early relaxation of this segment. (Reprinted with permission. ${ }^{\infty}$ )

dial edge that was visible when contrast perfused the myocardium (Fig 15). By subtracting the volume of the endocardial outline from the volume of the epicardial outline, an estimate of the volume of muscle could be obtained. Enddiastolic and end-systolic images were analyzed by videodensitometric techniques, and left ventricular muscle volume was compared to postmortem measurements. The end-systolic angiocardiographic estimate of muscle volume correlated best with the postmortem results $(r=.938$, standard error of the estimate $=5.9$ $\mathrm{mL}$ ). An extension of this method allowed experimental quantitation of myocardial perfusion
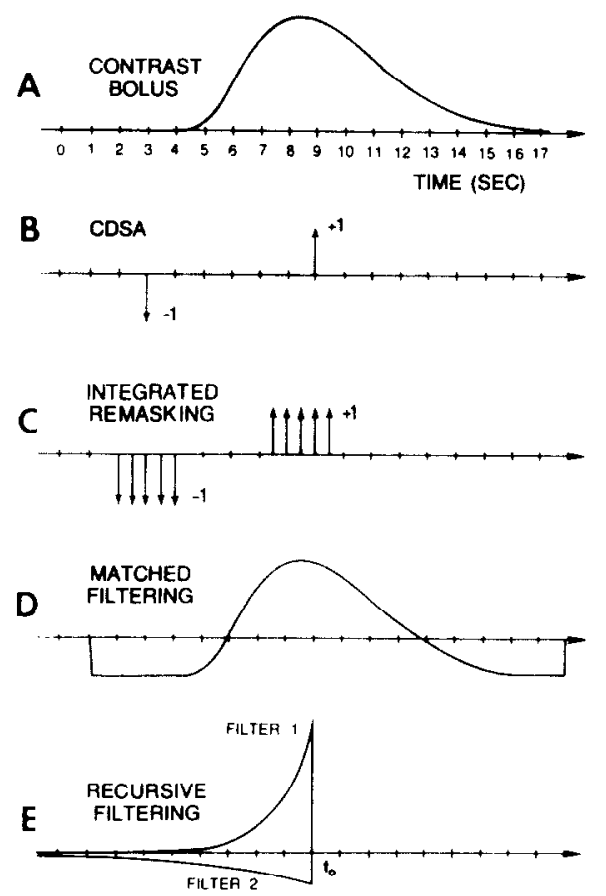

Fig 11. A schematic representation of alternate methods of temporal subtraction. (A) An idealized time $v$ intensity curve produced by an injection of contrast material as it traverses a specific region of interest. (B through E) Weights assigned to images acquired before and after contrast injection comparing this weighting for different temporal subtraction methods. The resultant image in each case is obtained by multiplying each original image by its respective weight and combining all weighted images together to effect the subtraction. CDSA. Conventional DSA (see figs 5, 6, and 7). (Reprinted with permission. ${ }^{68}$ )

deficits caused by coronary occlusion. In addition. measurement of free wall thickening from digital fluoroscopic images under control and ischemic conditions as well as during inotropic stimulation has been shown to accurately reflect regional thickening as validated with pairs of sonomicrometer crystals implanted across the myocardial wall in dogs. ${ }^{86}$

Gerber et al ${ }^{87}$ proposed an alternate method of quantitating regional function in a canine model of acute ischemia. Calculation of regional area displacement and the average amplitude of excursion of the anterior and posteroinferior walls were made from functional ejection shell and paradox images of digital IV ventriculograms. These images were produced by subtracting end-diastolic and end-systolic frames so that net inward motion or dyskinetic motion could be highlighted (Fig 16).

Digital IV ventriculography has been used in 

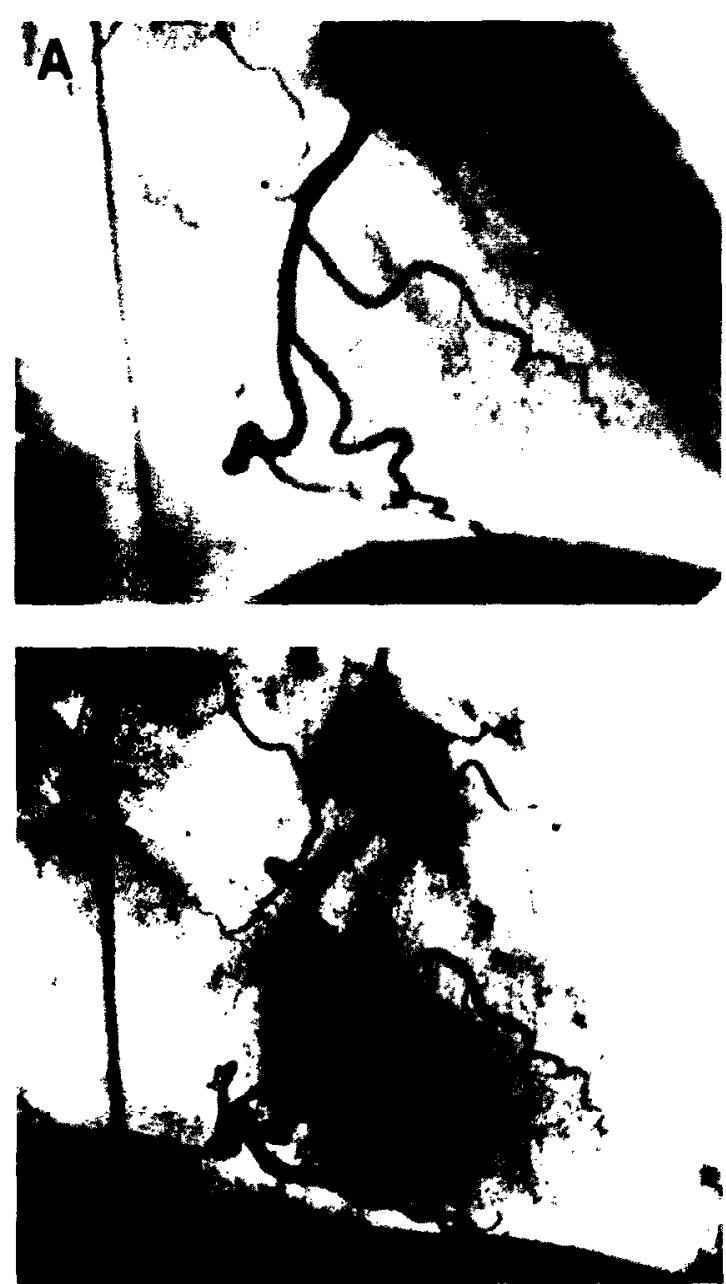

Fig 12. A normal selective right coronary angiogram as it appears using a temporal high-pass filter (A) and on the stenderd cine-view (B). (Reprinted with permission. ${ }^{\text {2 }}$ )

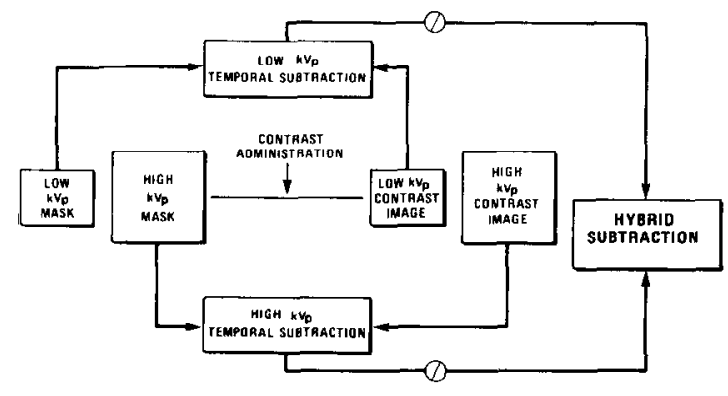

Fig 13. A schematic representation of the process of hybrid subtraction. (Reprinted with permission. ${ }^{74}$ )

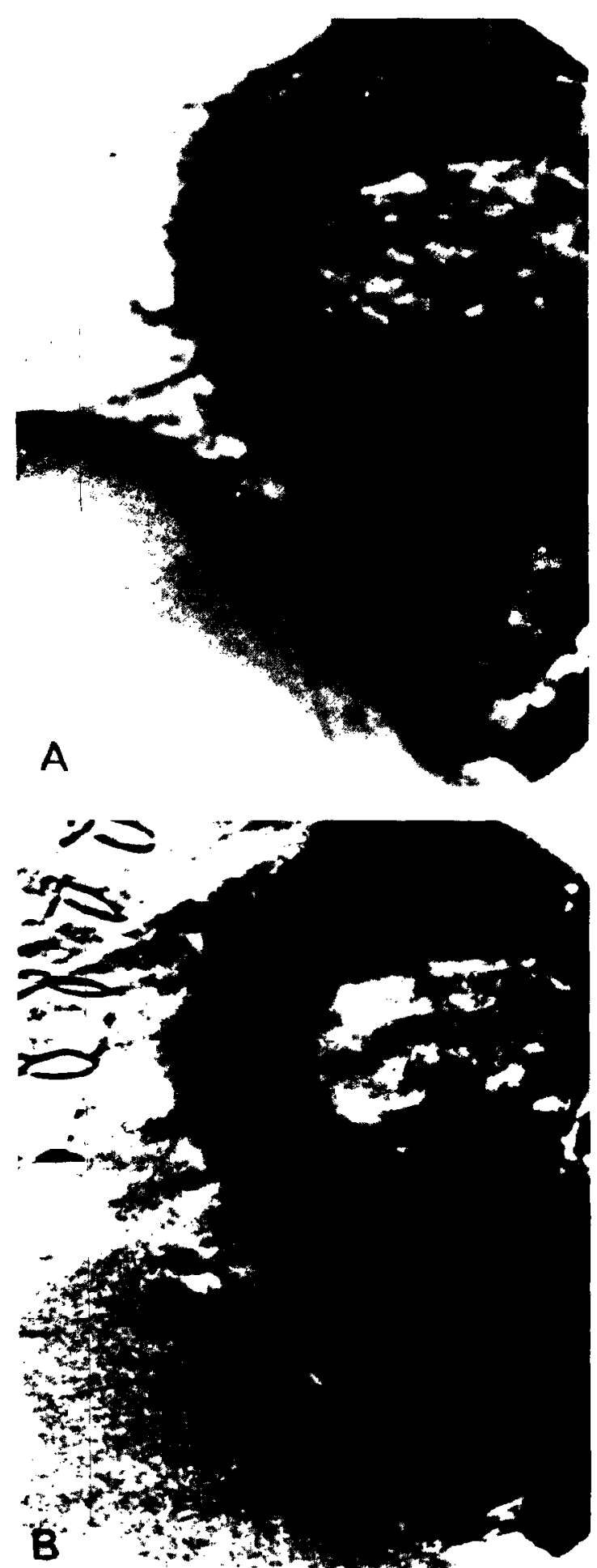

Fig 14. (A) An IV digital aortogram performed for the detection of coronary artery bypass graft patency. This was processed by mask-mode subtraction. (B) Improvement in the visualization of the bypass graft (arrow) is seen after hybrid subtraction. (Reprinted with permission. ${ }^{74}$ ) 


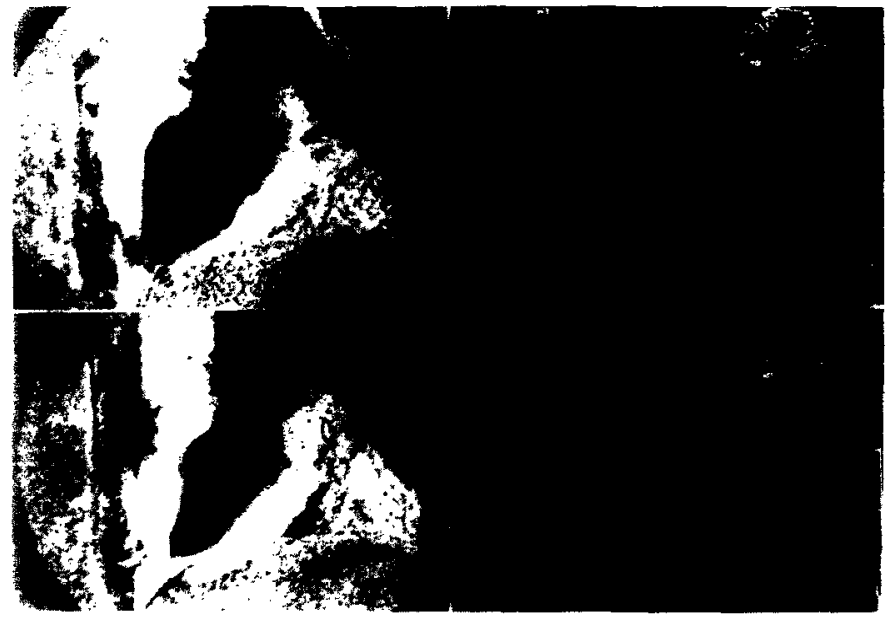

Fig 15. End-diastolic (upper left) and endsystolic (lower left) digital ventriculograms obtained in a dog in the left posterior oblique projection. Differential enhancement of the early (ventricular) and later (myocardial) phases of contrast injection allows excellent edge-definition of both endocardial and epicardial edges, allowing quantitative measurements of relative wall thickness (upper right) and estimates of wall thickening (lower right). animals to demonstrate the effects of acute incremental volume overload induced by surgically made arteriovenous shunts on cardiac chamber size and pulmonary transit time. ${ }^{88}$ During volume overload increases in both left and right ventricular end-diastolic volume, stroke volume and ejection fraction were demonstrated. These occurred in conjunction with an increase in cardiac output and a shortening of pulmonary transit times.

\section{Clinical Studies}

Digital IV ventriculography. Several studies have compared IV digital subtraction angiography and direct contrast left ventriculography to determine the accuracy and limitations of this technique and to detcrmine its potential clinical utility.

Vas et $\mathrm{al}^{89}$ examined volumes at end-diastole and end-systole and ejection fractions in four patients and noted similar results from the IV studies compared to conventional ventriculography except for a $2 \%$ to $7 \%$ systematic underestimation of volumes, probably related mainly to the subtraction and contrast enhancement process. In an extension of this work, ${ }^{90}$ ejection fraction determined by both techniques in 13 patients correlated with $r$ values between .752 and .854 , depending on the observer.

In a larger study, Tobis et al ${ }^{91}$ studied patients by both techniques separated by 24 hours. Enddiastolic volumes and ejection fractions correlated well ( $r=.82$ and $r=.96$, respectively) and without systematic errors. End-systolic volumes demonstrated a small systematic underestimation as well as an excellent correlation with conventional contrast measurements $(r=.93)$.

The comparative study of Kronenberg et $\mathrm{al}^{\text {th }}$ demonstrated high correlations with standard ventriculography $(r=.91$ and $r=.89$ for volume and ejection fraction data, respectively). Systematic underestimation of volumes was noted in the processed IV images but not in similarly processed images produced by low-dose intraven-

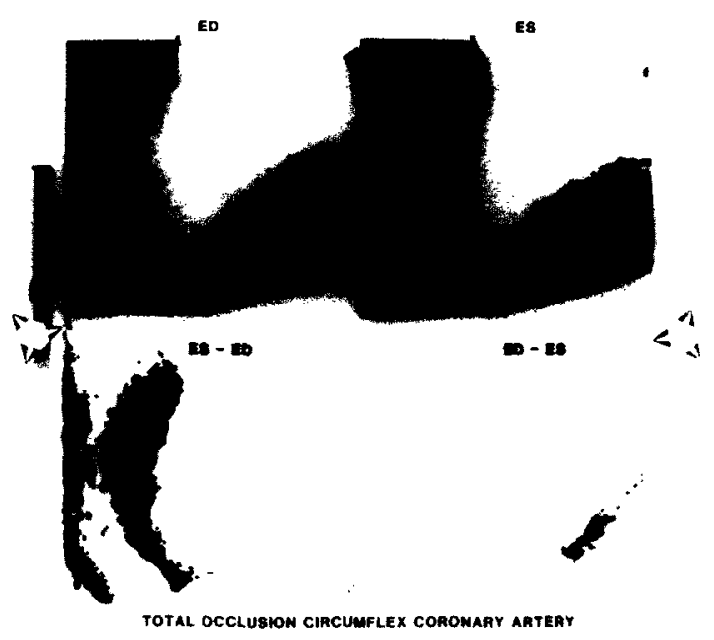

Fig 16. An IV digital left ventriculogram from a dog taken in the left posterior oblique projection and during total occlusion of the circumflex coronary artery. ED. End-diastolic frame; ES, end-systolic frame. ES minus ED results in an ejection shell image (lower left) which demonstrates enhanced function of the anterolateral wall and absence of inward inferior wall motion. ED minus ES results in a paradox image (lower right) that highlights the inferoposterior area of dyskinesis. (Reprinted with permission. ${ }^{\circ}$ ) 
tricular injection of contrast. The authors suggest that these differences may have been due to the fainter edge definition of the IV studies, and errors in estimating the location of the aortic and mitral valve planes which are obscured in IV ventriculograms.

Goldberg et al ${ }^{92}$ were uniformly successful in obtaining diagnostic images of the left ventricle, even in patients with ejection fractions of less than $30 \%$. End-diastolic and end-systolic volumes correlated extremely well ( $r=.96$ and .97 , respectively) as did ejection fraction determinations $(r=.98)$. It should be noted that other authors ${ }^{91}$ had difficulties in obtaining adequate left ventricular visualization in patients with very low ejection fractions, whereas some of this difficulty may have been overcome in the Goldberg study by use of a very rapid, central injection of contrast.

Norris et $\mathrm{al}^{93}$ demonstrated comparable correlative results, but volumes were systematically underestimated compared to direct left ventriculography (Fig 17). Again, these authors speculated that this finding represented a loss of sensitivity of edge detection caused by the smaller density differential between the diluted contrast nearest the endocardium compared to the soft tissue density of myocardial muscle.

A study by Felix et a ${ }^{94}$ included 46 patients, a large proportion of whom had poor left ventricular function. Although the direct ventriculograms and the IV ventriculograms were separated in time by a mean of three months, these investigators showed extremely high correlations

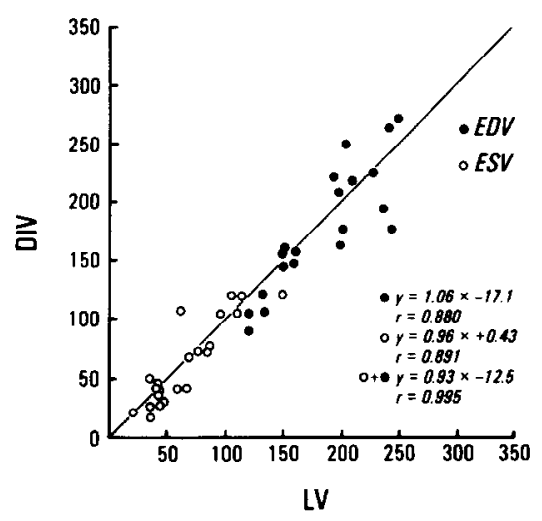

Fig 17. The correlation of end-diastolic volumes (EDV) and end-systolic volumes (ESV) obtained form standard cineventriculograms (LV) and digital intravenous ventriculograms (DIV). (Reprinted with permission. ${ }^{93}$ ) for ejection fraction $(r=938)$, end-diastolic volumes $(r=.979)$, and end-systolic volumes $(r=.925)$. They reported a systematic overestimation of end-systolic volumes when ejection fraction was below $36 \%$. This led to consistently lower ejection fraction results from the digital studies compared to the direct cineventriculograms. This finding was attributed to more homogeneous and complete opacification of the apical areas with the IV technique and the ability to enhance the contrast of the digital images. Thus, despite inclusion of a relatively large proportion of patients with depressed contractile function, no specific difficulties in imaging these patients were reported, and some improvement in quantitative analysis was seen in this study.

Nissen et al ${ }^{95}$ evaluated 40 patients, including 21 with prior myocardial infarctions. Similar results were demonstrated by this group (enddiastolic volumes, $r=.88$; end-systolic volumes, $r=.92$; ejection fraction, $r=.83$ ), and technically adequate studies were obtained even in patients with ejection fractions below $50 \%$. These investigators caution that persistent right ventricular or left atrial opacification in the setting of a low cardiac output or right ventricular failure can lead to difficulties in edge definition and quantitation in individual cases.

All of the previous investigations used maskmode image processing as the subtraction technique, whereas Engels et $\mathrm{al}^{96}$ reported ventricular assessments in 20 patients using TID processing of digital IV ventriculograms. Absolute volumes were not reported, and their study included only patients with ejection fractions greater than $50 \%$. Ejection fractions determined from the TID images correlated with direct left ventriculography with an $r$ value of .81 .

It is apparent from these studies that ventricular volumes and ejection fractions can be obtained from digital IV ventriculograms with an acceptable degree of accuracy to make the technique diagnostically useful. Furthermore, the successful study of patients with depressed ventricular function was reported by several groups. Although further investigation is needed, it seems these patients can be studied by this technique provided there is optimization of contrast injection, subtraction and enhancement techniques, and alternate views are consid- 
ered. ${ }^{78,97}$ Currently, however, it cannot be considered a procedure of choice in this subgroup of patients because of the potential deleterious effects of contrast media.

Low dose intra-ventricular digital angiography. For the reasons previously mentioned, a very attractive use of digital subtraction angiography is through direct, intraventricular injection of very small doses of contrast material. Diagnostic ventriculograms that provide accurate, quantitative information can be obtained without significant hemodynamic perturbations by subjecting these very faint images to processing with mask subtraction and contrast enhancement.

A dose of $5 \mathrm{~mL}$ of contrast material was used by Sasayama et $\mathrm{al}^{45}$ in 16 patients who also underwent conventional ventriculography. Using digital processing and an automatic edge detection algorithm, ${ }^{98}$ diagnostic images were obtained that demonstrated excellent correspondence to standard ventriculography $(r=.95$ and .98 for end-diastolic and end-systolic volumes, respectively). In order to ensure optimal mixing of blood and contrast, patients with large ventricles were excluded from the study, and the administration of contrast material was initiated by an electrocardiogram-triggered injection during the rapid filling phase of diastole.

Kronenberg et al ${ }^{46}$ used 5 to $10 \mathrm{~mL}$ injections of contrast material and compared ventricular volume measurements to conventional left ventriculograms in 12 patients. In the entire group of patients, a high correlation of volume measurements and ejection fractions from standard dose and low dose ventriculograms was demonstrated $(r-.96$ and .91 , respectively), but separate analyses of end-diastolic and end-systolic volumes were not provided

A different protocol was proposed by Nichols et $\mathrm{al}^{47}$ who used $7 \mathrm{ml}$. of ionic contrast material diluted in $43 \mathrm{~mL}$ of saline injected at $15 \mathrm{~mL} / \mathrm{s}$ for 3 seconds. Diagnostic studies were obtained even in patients with low ejection fractions and large ventricular volumes. The end-diastolic and endsystolic volume and ejection fraction correlation coefficients were all 0.97 . The same protocol was used by Seldin et al $^{99}$ who in addition used a semi-automatic border detection algorithm to obtain ventricular silhouettes from the digital studies. Again, high correlations were noted between low dose ventriculographic measurements and conventional ventriculography $(r=.94$ and .97 for end-diastolic and endsystolic volumes, respectively).

Tobis et $\mathrm{al}^{48}$ used $10 \mathrm{~mL}$ of contrast material because of inconsistent visualization with $5 \mathrm{~mL}$ injections. Although they were able to show only a modest correlation between conventional and low-dose ventriculograms in measuring enddiastolic volumes $(r=.77)$. end-systolic volumes were more accurately reflected $(r=.95)$.

Mancini and co-workers ${ }^{99 a}$ have recently extended these observations in a group of 31 patients studied by low dose digital ventriculography ( $10 \mathrm{~mL}$ of contrast in $10 \mathrm{~mL}$ of saline) and conventional ventriculography. Global parameters correlated well (end-diastolic volumes. $r=0.85$; end-systolic volumes, $r=0.93$; ejection fractions, $r=0.92$ )

Despite the different low dose injection protocols mentioned above, all groups showed the expected lack of significant hemodynamic perturbations in response to low-dose direct digital ventriculography. This application allows safer examinations of patients with pre-existing renal disease, diabetes, multiple myeloma, aortic stenosis, unstable angina, or poor ventricular function (Fig 18). Furthermore, as has been demonstrated. ${ }^{100}$ ventriculography can be safely repeated to assess the effects on ventricular function of interventions such as atrial pacing or to obtain sequential, orthogonal views of the ventricle in the absence of biplane angiographic facilities.

It should be appreciated that direct intraventricular injection of contrast materials, whether diluted or not, will lead to streaming and inhomogeneous opacification in some instances that may cause indistinct visualization of the ventricular margins. Inhomogeneity of opacification also occurs as noncontrasted blood flows into the ventricle through the mitral valve. In addition to this, regional wall motion abnormalities can, on occasion, preclude adequate regional ventricular mixing of contrast. This can aggravate the problem of contrast streaming if an insufficient volume of contrast material is used. From analysis of the previously cited studies and in the authors" experience, ${ }^{99_{a}, 101}$ the use of diluted doses of contrast material compared to undiluted doses more consistently provides images of diagnostic quality in a broad spectrum of patients. 
Fig 18. A direct left ventriculogram obtained with $10 \mathrm{~mL}$ of contrast medium diluted in $10 \mathrm{~mL}$ of saline and injected over 2 seconds in a young diabetic patient with post-viral cardiomyopathy. The end-diastolic frame is shown in the upper left panel and the end-systolic frame in the lower left. Quantitative parameters in the right panels demonstrate severe global dysfunction, an ejection fraction of $4 \%$ and markedly increased ventricular volumes. Despite a resting puimonary wedge pressure of $30 \mathrm{~mm} \mathrm{Hg}$, this diagnostic ventriculogram was obtained without any major hemodynamic perturbations or patient discomfort.
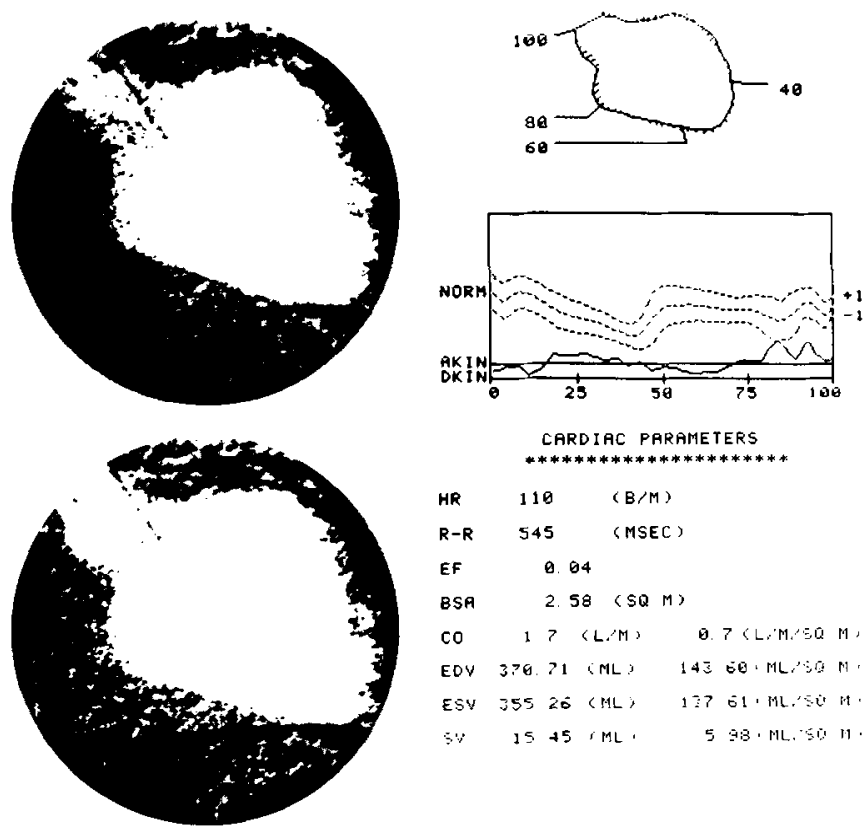

Assessment of global ventricular function with densitometry. It is beyond the scope of this report to discuss the technical features, constraints, and limitations of densitometry as it applies to cardiac imaging in humans. It is apparent that digital cardiac imaging can provide not only geometric information that allows, for example, area-length calculations of ventricular volumes but can in addition give information about the depth or volume of heart chambers and vascular structures by densitometry. The attraction of this methodology is in the circumvention of geometric assumptions required to make volume measurements (a feature that limits traditional geometric methods, particularly in the setting of wall motion abnormalities), and when attempting to characterize chambers that are not easily described by geometric shapes, such as the right ventricle. Thus, with appropriate processing of the transmitted $x$-ray signals, the digitized grey scale values making up an image are theoretically linearly proportional to the amount of contrast traversed by the x-ray beam. With accurate calibration and homogeneous mixing of contrast, non-geometric measurements of absolute or relative volumes are possible. This approach has been used to determine ventricular volumes and function, and accurate results have been reported..$^{10,81,102-108}$ It must be appreciated, however, that the relationship between $\mathrm{x}$-ray beam attenuation and contrast depth is affected by several physical properties of roentgen imaging that require complex corrections. ${ }^{109-113}$ Advances in the ability to correct these errors will result in powerful quantitative abilities for digital cardiac imaging because the excellent spatial information could then be combined with volumetric data.

Assessment of regional ventricular function. The interobserver variability of subjective assessments of regional wall motion is known to be quite marked..$^{90.114}$ Digital ventriculography is well suited to rapid and routine quantitative analyses of segmental function that can reduce interobserver and intraobserver error, and this is of particular importance if interventions are to be accurately studied. Despite this, relatively few studies have used quantitative techniques to compare conventional and digital subtraction ventriculography in the assessment of regional wall motion.

$V$ as et al ${ }^{90}$ emphasized the poor reproducibility of subjective wall motion assessment from either conventional left ventriculograms or contrast-enhanced IV digital ventriculograms. With the use of an automated radial analysis method to quantitate regional function, interobserver variability was minimized.

Low contrast-dose, intraventricular digital left ventriculograms and standard ventriculograms 
were compared on a regional basis in 28 patients by Nichols et al. ${ }^{47}$ They measured percent chordal shortening of hemiaxes and showed a moderately good correlation $(r=.81)$ of results that may have been impaired by an effective framing rate of only ten per second.

Mancini et al ${ }^{115}$ used a radial shortening technique to assess concordance in regional wall motion analysis. In contrast to the prior study, this study used digital IV ventriculography. With this technique, the left atrium and basal portions of the left ventricle and the aorta were simultaneously opacified, and the usual landmarks for assignment of the major axis of the ventricle were obscured. Thus, the long axis of the digital ventriculogram was taken as the line joining the ventricular apex to the centroid of the enddiastolic frame, whereas the long axis of the direct ventriculogram was taken from the midpoint of the aortic valve plane to the apex. As a result, normal ranges of wall motion for these two imaging techniques were systematically different. However, by using the quantitative criteria appropriate to each image, agreement in assessing normal and abnormal motion was found in $87 \%$ of segments. As expected, most disparities occurred in the basilar and apical radii which are known to be quite variable, are sensitive to assignment of the long axis, and which are obscured (basal regions) in the digital IV ventriculograms.

Mancini et al ${ }^{99 a}$ extended this work by quantitative measurement of regional wall motion from low contrast-dose ventriculograms compared to standard ventriculograms. In contrast to the study of Nichols et al, ${ }^{47}$ this study used a framing rate of thirty per second thus enhancing the accuracy of regional function measurement. Under these conditions quantitative regional function by both techniques correlated well $(r=0.90)$.

Using a regional area reduction method for objective wall motion. Nissen et $\mathrm{al}^{95}$ demonstrated a high concordance rate $(91 \%)$ in classification of wall motion from IV digital ventriculograms and direct ventriculograms.

Engels et al $^{96}$ analyzed TID ventriculographic images and found a high correlation with standard ventriculography in quantitation of anterior ( $r=.89$ ) but not inferior $(r=.62)$ wall motion. This problem was attributed to diaphragmatic overlap in the inferior regions. It should be noted that the specific method of wall motion assessment was not described in this study.

None of the geometric approaches to wall motion measurement are universally accepted because of known limitations of each technique. ${ }^{116-119}$ Widmann et al ${ }^{120.121}$ described an alternative technique of regional function analysis by using a Fourier analysis of the x-ray transmission $v$ time curve for each pixel making up the left ventricular region of digital IV ventriculograms. The first harmonic curve of the Fourier analysis could be quantitated on the basis of curve amplitude and phase angle. These parameters give information regarding the extent and synchronicity of dynamic wall motion relative to the other pixels. Histograms of amplitude or phase angle $v$ number of pixels can then be generated and quantitated. For example, a large standard deviation of the histogram relating phase angle $v$ number of pixels is a reflection of asynchronous wall motion, whereas a small standard deviation indicates relatively synchronous motion. Using this approach, they were able to demonstrate greater asynchrony in ischemic compared to normal ventricular segments (Figs 19 and 20).

Ventricular function in response to ischemic stress. Mask-mode digital ventriculography does not lend itself readily to assessment of ventricular function during exercise because of patient motion and consequent misregistration artifacts. In addition, breath holding during or after exercise is difficult. Atrial pacing, which obviates these problems, has been used by several groups to induce global and regional ischemic dysfunction in patients with coronary disease. Twenty-one patients studied by Tobis et al ${ }^{(0)}$ were challenged with rapid atrial pacing. I ow contrast dose, direct digital ventriculograms were analyzed at rest and during the peak phase of pacing, Fourteen of 15 patients (93\%) with significant, anatomical coronary disease showed a decrease or no change in ejection fraction during pacing, whereas five of six patients with chest pain syndromes and anatomically normal coronary arteries showed an increase of $5 \%$ or more in this parameter. Qualitative assessment of regional wall motion demonstrated new or worsened abnormalities in 12 of the 15 coronary patients $(80 \%)$. 


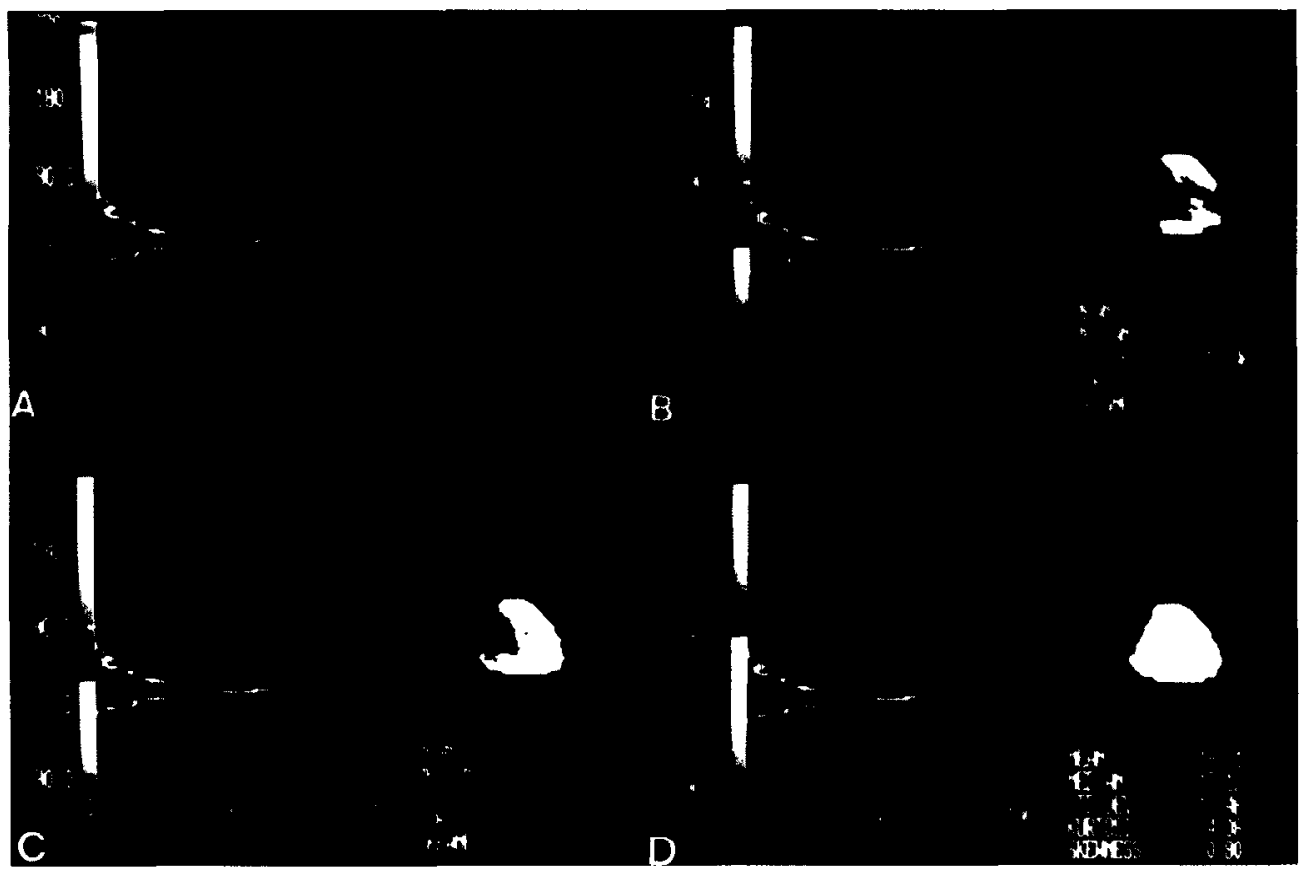

Fig 19. Phese image and quantitative phase histogram analysis in a normal patient. Images correspond to an area of interest at end-diastole (A), early systole (B), mid-systole (C), and end-systole (D). Contraction is synchronous in the various left ventricular regions accounting for a narrow base (standard deviation) and peak of the phase angle histogram. (Reprinted with permission. ${ }^{122}$ )

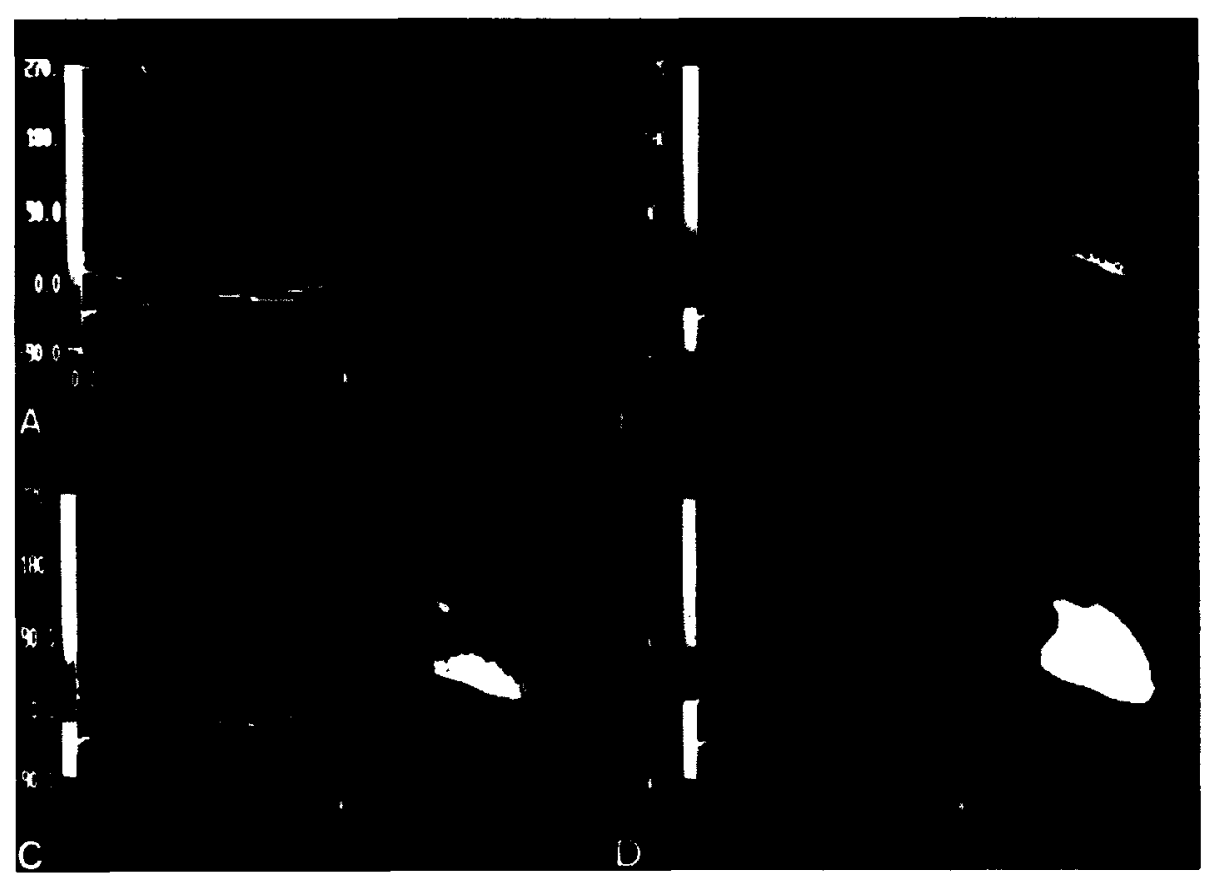

Fig 20. Phase image and quantitative phase histogram analysis in a patient with a high-grade stenosis of the left anterior descending coronary artery. The format is the same as Fig 19. There is delayed onset of contraction in the enterior wall accounting for a broad base and peak of the phase angle histogram. (Reprinted with permission. ${ }^{122}$ ) 
Mancini et al ${ }^{123}$ performed a similar study in 22 patients referred for cardiac catheterization. Differences compared to the prior study include the use of IV digital ventriculography, analyses during the post-pacing phase of rapid atrial pacing stress and the use of quantitative criteria to assess wall motion. The post-pacing phase was chosen for analysis so that global and regional parameters could be assessed at similar heart rates and when inotropic effects of tachycardia had dissipated. ${ }^{124-129}$ Coronary patients showed no overall change in post-pacing end-diastolic volumes but a significant increase in end-systolic volumes and a fall in ejection fraction were detected (Fig 21). In addition, quantitative deterioration of segmental wall motion was seen in $82 \%$ of coronary patients and in $91 \%$ of a subgroup of coronary patients who had totally normal resting wall motion (Fig 22). These perturbations of global and regional function were not seen in the normal group.

Johnson et al ${ }^{130}$ also used digital IV ventriculography in conjunction with rapid atrial pacing, but pacing was used to evoke regional dysfunction only in patients shown to have normal resting wall motion. As in the previous study, regional analyses were made in the early postpacing phases of the atrial pacing stress. How-

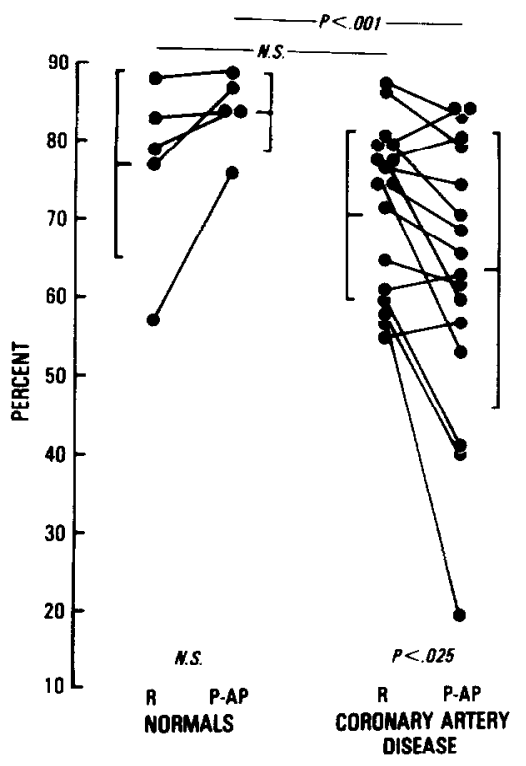

Fig 21. Ejection fraction at rest and after atrial pacing in normal subjects and patients with coronary artery disease as assessed by digital IV ventriculography. R, rest; P-P. post-pacing. (Reprinted with permission. ${ }^{123}$ )
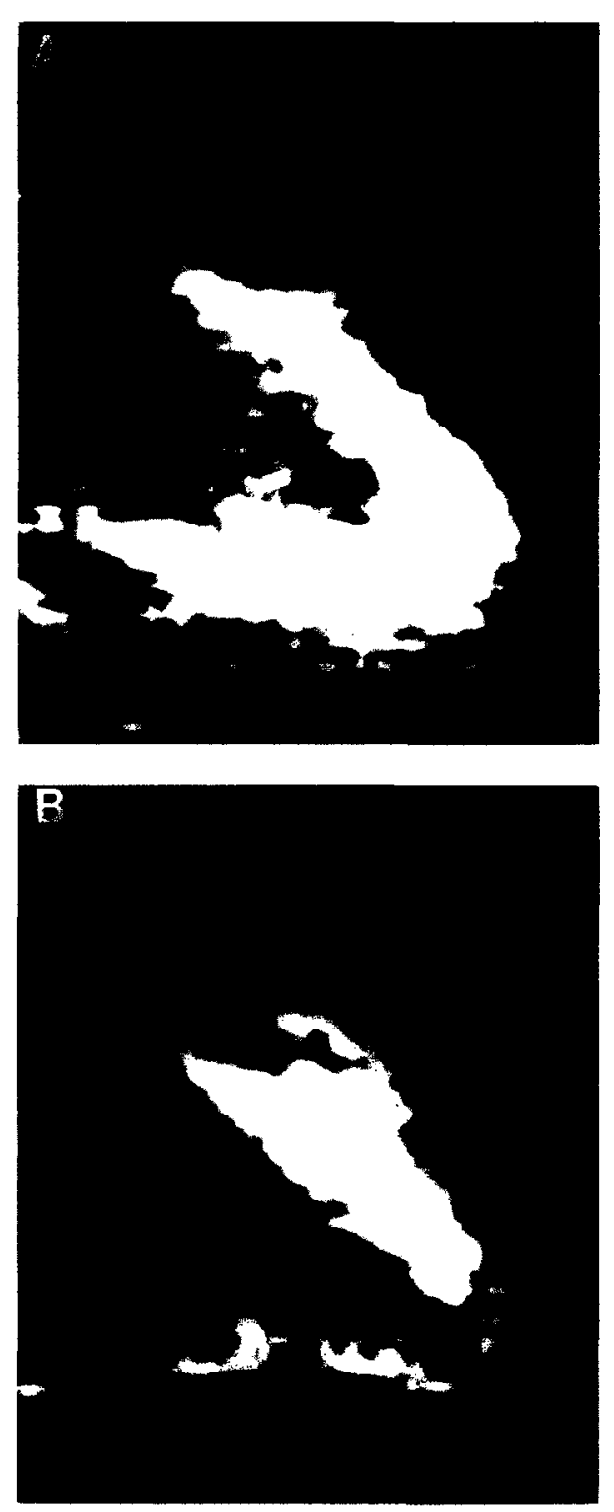

Fig 22. The ejection shell images of a coronary patient in the control state (A) and after atrial pacing (B). The ejection shell is obtained by subtracting the end-systolic frame from the end-diastolic frame, leeving a white ejection shell. At rest, normal endocardial excursion is indicated by a uniform, white ejection shell in all ventricular regions, whereas after pacing a new area of akinesis is present as evidenced by the absence of a white rim in the inferior region. The ventricle was imaged in the $30^{\circ}$ right anterior oblique projection. (Reprinted with permission. ${ }^{123}$ )

ever, subjective wall motion grading was used, and an analysis of volume and ejection fractions was not provided. Nevertheless, of 16 patients with normal coronary arteries and normal wall motion at rest, none developed a post-pacing regional abnormality. In contrast, of 34 patients 
with anatomical coronary stenoses and normal resting function, $28(82 \%)$ demonstrated a new segmental abnormality after atrial pacing.

These studies highlight the usefulness of this technique in providing a sensitive and specific means of diagnosing coronary disease or in demonstrating the functional significance of coronary lesions when regional wall motion is assessed after an ischemic stress. Although the low-dose contrast method minimizes the use of contrast material at the time of cardiac catheterization, the IV techniques are more suitable for precatheterization screening of patients. Differences in the peak- and post-pacing determinations of ventricular function are likely to have effects on the sensitivity and specificity of this form of stress testing, as will the method of wall motion analysis, particularly if subjective methods are used. For these reasons, efficient, standardized protocols and quantitative criteria, similar to current standards of exercise testing, must be established for optimal clinical use of this form of stress testing.

Patient motion and imperfect breath holding are significant, practical limitations to the use of mask-mode digital subtraction angiography in assessing ventricular function during or immediately after exercise. Nevertheless, several preliminary reports have suggested that diagnostic images can be obtained in the majority of cases with exercise digital IV ventriculography. ${ }^{131-134}$ Goldberg et $a l^{133}$ reported their experience with this application in 31 paticnts. In only two instances were the exercise ventriculograms degraded to such an extent that they were nondiagnostic. These instances occurred in the early phase of the study when the investigators had not yet determined the appropriate timing of breath holding during exercise. With continued experience, diagnostic studies were obtained routinely. Ejection fraction changes were similar to those seen during exercise radionuclide angiography and all patients with significant coronary disease manifested new or worsened wall motion abnormalities. This study demonstrates that excercise digital IV ventriculography can be used effectively provided that meticulous attention is paid to the details of coordinating the cessation of inspiration with the passage of contrast material from the right to the left heart during exercise. These constraints may conceivably be mitigated by reregistration programs, respiratory gating or alternate image processing techniques, but these innovations may make clinical implementation even more difficult. Currently, exercise testing with radionuclide angiography is more truly noninvasive, is not heavily degraded by respiratory motion, involves less patient discomfort, and less physician interaction. Because the diagnostic precision is not importantly improved by the digital radiographic techniques, it seems to have little current advantage over rest-exercise radionuclide ventriculography for the detection of coronary disease, but the greater spatial resolution may offer some advantage in assessment of regional function.

Assessment of left ventricular wall thickness. Wall thickness and wall thickening dynamics are extremely important in the comprehensive characterization of left ventricular function. High quality cineangiograms or video images have previously been used to obtain an estimate of left ventricular wall thickness, muscle mass, and thickening characteristics. ${ }^{135-137}$ These approaches have been limited by several practical problems that include (1) loss of accurate endocardial edge definition, particularly at end-systole; (2) inconsistent epicardial definition; (3) uncertainties in the measurement of true wall thickness from radiographic silhouettes of endocardial and epicardial edges; (4) difficulties in accurately tracking comparable segments throughout the cardiac cycle, a difficulty caused by complcx translational and rotational wall motion; and (5) a lack of generally available, automated methods of analysis. Despite these limitations, the use of such measurements to provide an index of wall thickness and thickening has been shown to be of value in characterizing ischemic segments, ${ }^{136,138,139}$ the effects of bypass grafts on regional function, ${ }^{140}$ regional alterations of function in cardiomyopathies, ${ }^{141}$ and in measuring left ventricular tension and stress. ${ }^{142-145}$ Current digital imaging is capable of making these measurements more generally available and on a routine, automated basis. Currently, endocardial edge definition is adequately provided by the IV and intraventricular applications of digital ventriculography mentioned previously. Although more difficult to ascertain, epicardial edge definition has been augmented by several image processing methods 
including TID imaging ${ }^{96.146}$ or ECG-gated frame integration to enhance the myocardial perfusion of contrast material after IV, intraventricular, or aortic root inejction ${ }^{84.85}$ (Fig 15). Further investigation of these and other methods are required to determine the role of digital subtraction angiography in this area.

\section{ASSESSMENT OF CORONARY ARTERIES AND SAPHENOUS VEIN BYPASS GRAFTS WITH DIGITAL SUBTRACTION ANGIOGRAPHY}

Coronary arteriography is currently considered the "gold standard" by which the extent of coronary disease is defined. Although of revolutionary diagnostic importance, after 20 years of implementation, the assessment of coronary angiograms is still fraught with technical problems, significant intra- and inter-observer variability in stenosis quantitation. ${ }^{147-152}$ and lack of correlation with anatomical findings. ${ }^{153-155}$ The ability to ascribe physiological significance to specific coronary lesions, regardless of how accurately the percent stenosis is defined, has recently been seriously questioned. ${ }^{156-158}$ Digital subtraction angiography offers specific advantages that can help overcome some of the limitations of standard coronary cineangiography. As with digital subtraction ventriculography, these advantages result from the ability to manipulate images by subtraction and contrast enhancement, the ability to easily quantitate images because of the inherent digital data format, and the potcntial to extract, in a practical way, new information from coronary images, such as intensity (or density) and contrast transit parameters that may be of diagnostic value in the clinical evaluation of coronary disease. A full exploitation of these characteristics is in the early stages of experimentation and clinical application.

It is worth emphasizing, however, that much of the initial clinical enthusiasm for this technology was due to the prospects of imaging the coronary arteries by an IV contrast injection. Unfortunately, this has been the most intriguing. but least successful, application of digital IV angiography, even in very sophisticated laboratories. ${ }^{18,77,112}$ Investigators at the University of Wisconsin have recently outlined some of the problems that have thwarted this effort. ${ }^{77,112}$ These include the limitations imposed by overlying iodinated pulmonary and cardiac structures. large transmission dynamic range, vessel motion, and motion of noniodinated structures. They report that the problem of overlying contrastladen pulmonary and cardiac structures can be suppressed with the use of high pass filtration techniques. The trade off. however, is that enhancement of background structures similar in size to the coronary vessels may occur. The problem of large transmission dynamic range refers to the cross scatter from bright lung fields that can decrease contrast behind the adjacent. darker regions of the opacified ventricle. The resultant inhomogeneities in the distribution of the information content across the radiographic image may be overcome with further development of a proposed, digitally controlled beam attenuator which allows selective $x$-ray exposure to various portions of the image field. ${ }^{77,112,159}$ Both respiratory and cardiac motion can cause significant degradation of the subtracted coronary images. Although this group found that the use of a phase matched subtraction method using separate mask images for each portion of the cardiac cycle did not appear to improve vessel visualization, other groups ${ }^{160}$ have reported that this type of phase coordinated subtraction is crucial for adequate imaging of the coronaries. Other subtraction techniques, such as hybrid subtraction, may be useful in visualizing coronary arteries or bypass grafts by eliminating soft tissue misregistration artifacts. ${ }^{99.74,11 ?}$ (Fig 14). Despite potential technical advances, it is doubtful that the proximal and distal portions of the coronary arteries can be routinely and reliably visualized by IV techniques. It is frequently necessary to obtain different angulated views to properly unravel complex coronary anatomy, and such repeated studies are severely limited with IV angiography because of the excessive contrast doses required.

Nevertheless, IV digital techniques currently do have limited roles in the assessment of bypass graft patency. Myerowitz et al ${ }^{161-163}$ report that out of 26 saphenous bypass vein grafts assessed by both mask-mode digital IV angiography and selective angiography, 11 grafts were noted to be occluded on both studies, and 11 out of 15 truly patent grafts were correctly identified from the IV studies (sensitivity of $73 \%$ and specificity of $100 \%$ for the detection of patent grafts). Drury et $\mathrm{al}^{164}$ reported a $69 \%$ sensitivity for detection of 
patent bypass grafts and $100 \%$ specificity when IV techniques were used. The low sensitivity of this technique in detecting patent grafts is partially related to the inability to detect grafts that overlap the opacified aorta and ventricle. Thus, multiple views, within reason, may yield better results. However, detailed information about proximal or distal anastomoses and distal runoff is currently not provided by this technique. As mentioned above, alternate masking or subtraction techniques may further improve graft visualization. ${ }^{74,77,112}$ But contrast-enhanced computed tomography is currently superior in assessing bypass patency ${ }^{165,166}$ and is preferable over IV digital subtraction methods.

Alternatively, digital imaging with aortic root injection of small or diluted doses of contrast material has been used to assess bypass graft patency. Two groups ${ }^{164.167}$ have reported $100 \%$ sensitivity and specificity in detecting graft patency by this technique. Details of anastomotic sites are again not accurately provided, but information as to whether the grafts are patent or not can expedite selective catheterization of the grafts and obviate repeated radiation exposure and contrast injections in searching for grafts that might actually be occluded (Fig 23). This is particularly advantageous when studying patients who do not have metallic graft markers to guide the angiographer. Furthermore, with the use of small or diluted doses of contrast material, repeated aortography can be performed to overcome some of the problem of overlap.
Anatomical assessment of native coronary arteries has similarly met with limited success when aortic root injections are used. ${ }^{167.168}$ On the other hand, there are definite advantages to be gained by applying digital techniques to selective coronary angiography.

Several studies have underscored the immense degree of interobserver and intraobserver variability in determining the percent coronary stenoses from routine cineangiography. ${ }^{147-152}$ The value of even simple quantitative techniques in reducing this marked degree of variability has been shown by several groups. ${ }^{169-174}$ Despite this, the quantitative methods are somewhat time consuming and potentially inaccurate due to geometric assumptions about lesion shape. These are compounded by the problems of radiographic imaging, such as pincushion distortion, differential magnification, and quantum mottle. ${ }^{169,171,175,176}$ In addition, the percent stenosis measurement is based on a comparison of the stenotic area to a presumed normal segment which may indeed not be normal. This relative measurement may seriously misrepresent the absolute degree of luminal encroachment, and the potential effects of the length of the lesion or serial lesions are often not taken into account. ${ }^{177,178}$

Brown et a $1^{175}$ described a computer assisted method for analyzing coronary angiograms that takes into account potential effects of pincushion distortion and differential magnification, and provides quantitation of lesion lengths, diame-
Fig 23. A direct, digital aortogram obtained with $20 \mathrm{~mL}$ of a $50 \%$ solution of contrast material. This patient had aortocoronary bypass grafts to the left anterior descending and first diagonal branches of the left coronary artery. Notice that although the proximal segments are clearly seen, no definite anatomical information about the anestomoses or dista vessels are available from this image. In addition, the main bodies of the grafts are overlapped in this projection. Despite these limitations, selective catheterizetion of grafts can be expedited by first screening for graft patency by this technique.

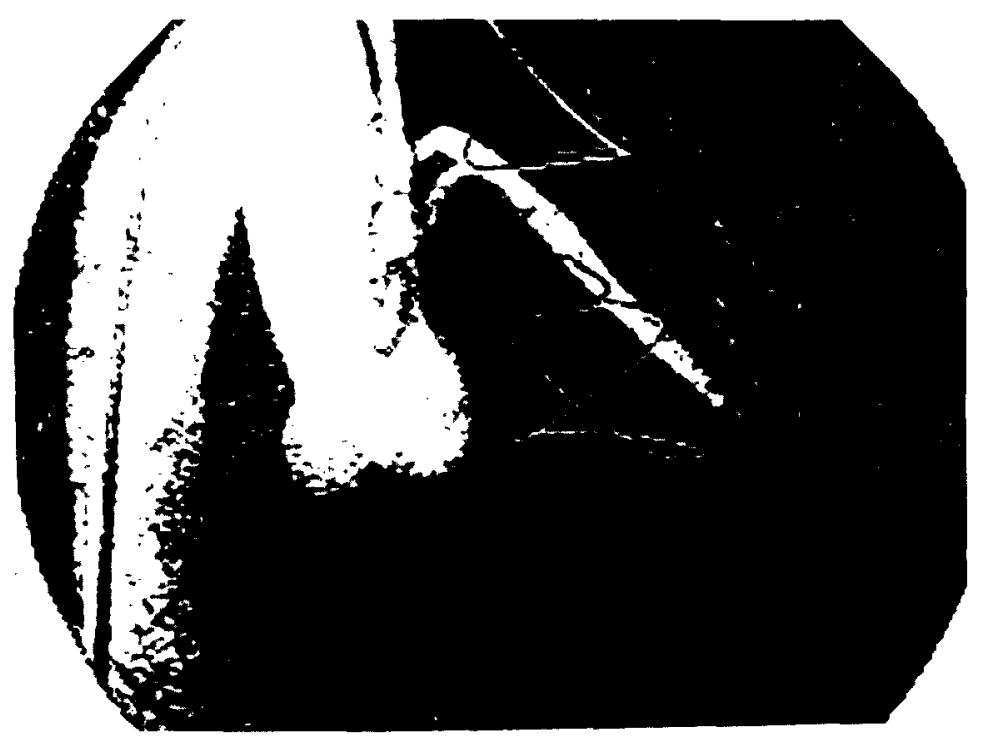


ters, and cross sectional areas. While of definite importance, the computation time required to analyze two perpendicular views of each coronary stenosis is substantial and the method relies on operator defined edges. Several authors have proposed various edge-detection algorithms that are suitable for analysis of coronary angiograms. ${ }^{179-182}$ These techniques are useful in minimizing subjective aspects of edge-definition by using strictly defined edge-parameters. Furthermore, these techniques can be readily adapted to on-line digital coronary angiography, eliminating cumbersome digitization of cineangiographic frames.

Although these innovations can increase the accuracy of anatomically defining coronary lesions, Spears et al ${ }^{183}$ point out that significant errors in estimating the luminal area of an elliptically-shaped coronary stenosis can occur when only two orthogonal views are analyzed, even under ideal conditions of edge-detection. This error is relatively small for stenoses with only mild degrees of ellipticity, but progressively larger errors can result when lesions are more elliptical. Theoretically, densitometric analyses of coronary stenoses circumvent the geometric limitations of the measurements outlined above, and this has been demonstrated by several groups. ${ }^{184-189}$ Thus, the methods outlined. whether geometrically or densitometrically based, and with or without edge-detection algorithms, are all ideally suited to analysis of digitally acquired and processed coronary angiograms obtained by selective contrast injection, especially if the process is on-line, eliminating cumbersome digitization of cineangiograms. It is conceivable that the densitometric methods will decrease the need for multiple, high frame rate, angiographic views. These properties of digital coronary angiography may compensate to a certain degree for the inferior spatial resolution of digital angiograms compared to cineangiograms. ${ }^{188}$

Although the above methods can facilitate the quantitation of coronary stenoses and reduce interobserver and intraobserver variability, the ability to discern the physiologic importance of coronary lesions from their mere anatomical appearance is profoundly limited, particularly when stenoses are of intermediate anatomical severity. ${ }^{156-158}$ For example, using both videomet- ric analysis and quantitative, geometric methods, investigators at the University of Iowa showed only modest correlations between these measurements and determinations of postocclusion reactive hyperemia. ${ }^{156}$ More recently, this group has shown that to a certain extent, measurement of minimal cross-sectional area of proximal left anterior descending stenoses can be used to predict impairments of coronary flow reserve. ${ }^{158}$ Thus, another more direct approach to the use of digital coronary angiography has been to extract information about actual coronary flow and myocardial perfusion dynamics to attempt to predict the potential functional consequences of coronary lesions, irrespective of their anatomical morphology.

Much of the current work in the area of arterial flow assessments with digital angiography is based on the techniques explored at the University of Zurich and the Mayo Clinic. ${ }^{190-193}$

Rutishauser et a ${ }^{190,191}$ used roentgen densitometry to measure mean transit time of a contrast bolus in epicardial segments of coronary arteries. Then, by geometrically measuring the length and diameter of the coronary segment, an estimate of coronary flow could be calculated. This method accurately reflected epicardial blood flow measured simultaneously with an electromagnetic flowmeter in dogs. With similar techniques, Smith et al ${ }^{192.193}$ measured coronary flow and saphenous vein bypass graft flow after aortic root injection of contrast materials. These methods are limited to nonbranching arterial segments and by the accuracy with which coronary dimensions can be measured from single or biplane angiography and the accuracy with which transit times can be determined from videodensity curves. Smith et al emphasized the need for correction of videodensity background signals due to vessel motion and changes in cardiac size during the heart cycle. They also pointed out the alteration of the usual indicatordilution curve (prolonged downslope) due to persistence of contrast material in the myocardium and recirculation in the cardiac chambers superimposed on the regions of interest of the coronary segments. The validity of the measurement also rests on the presupposition that contrast material itself does not substantially alter coronary flow. Although this was true of the aortic root injections of small amounts of contrast, more signifi- 
cant alterations of flow were noted by Rutishauser et al when selective coronary injections were used. Early changes in coronary blood flow after selective ionic contrast injection have recently been shown to be substantial. ${ }^{194}$

Spiller et a $1^{195}$ have extended and modified this approach to obtain systolic and diastolic blood flow measurements in native coronary arteries and saphenous vein bypass grafts in man. Cineangiograms were obtained in patients at the time of bypass grafting while simultaneous electromagnetic flow determinations were recorded. Small doses of ionic contrast were selectively injected by an ECG-gated power injector. The angiograms were processed and analyzed by densitometric methods to produce densograms at two sites over vessels and the velocity of contrast movement was determined from the leading slopes (front velocities) of background-subtracted densograms at the point where the density reached one half of its maximal value. Velocity measurements were converted to flow measurements by determining the length and width of the vessel segment between the sampling sites. Repeated contrast injections during different phases of the cardiac cycle allowed determination of phasic coronary blood flow. Excellent correlations between densitometric flow and electromagnetic flow determinations were reported $(r=.97)$ with a systematic overestimation of approximately $20 \%$ that was ascribed to the use of the front velocities instead of mean transit times (Fig 24). Despite this overestimation, the authors present arguments as to why the front velocity method is preferable to mean transit time measurements for flow determinations. In essence, this is because mean appearance times are determined from the entire densogram and the end portions of these curves are known to be altered by layering and delayed washout of contrast, factors which do not affect the appearance of the front of the contrast bolus. Furthermore, mean transit time measurements preclude determination of pulsatile flow. Another advantage of this method is that the determinations were made very early after contrast injection, within $650 \mathrm{~ms}$, when major changes in blood flow caused by contrast injection have not yet occurred. ${ }^{194}$ Despite these advantages, the technique is still limited by the accuracy with which short epicardial vessel segments can be mea-

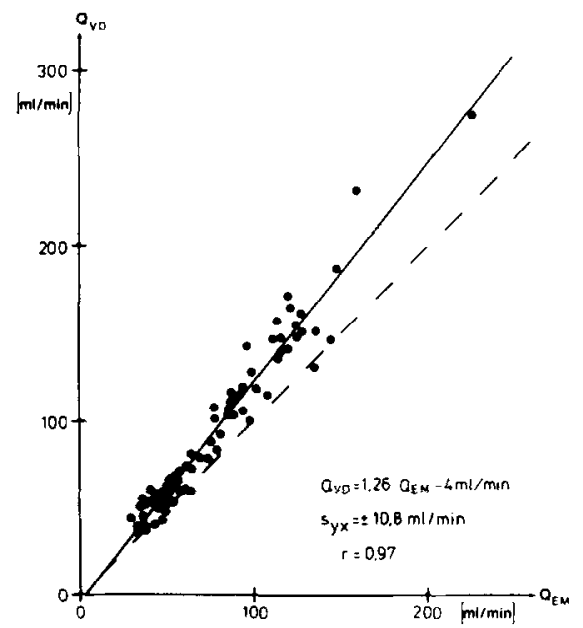

Fig 24. Relation between videodensitometrically determined flow $\left(Q_{\mathrm{vD}}\right)$ and the electromagnetically determined flow $\left(Q_{\mathrm{EM}}\right)$ in aortocoronary bypass grafts measured at the time of operation. The electromagnetically determined flow is overestimated by about $20 \%$ by the videodensitometric method. Solid line, regression line; dashed line. line of identity. (Reprinted by permission of the Americen Heart Association. Inc from Spiller P. Schmiel FK, Politz B. et al: Circulation 68:337-347, 1983. ${ }^{196}$

sured, the need for repeated injections during different phases of the cardiac cycle, and the laborious processing required.

Another approach to the functional assessment of coronary flow has been to determine relative coronary flow during control conditions and during contrast-induced hyperemic flow. Determination of impaired reactive hyperemia has been shown by Gould et al ${ }^{196.197}$ to be a sensitive indicator of the functional significance of coronary stenoses.

Foerster et al ${ }^{198.199}$ used videodilution techniques to measure this relative index independent of geometric measurements of coronary segments. In their series of investigations, a theoretical equation for determination of relative flow was derived, taking into account not only the high response characteristics of videodensitometers used in prior studies to measure transit time, but also the relationship between the videodensity amplitude response, and the mass of contrast material traversing the region of interest over the coronary artery. The videodensitometer output was directly related to the mass of contrast medium in the vessel segment and a constant parameter derived from the effects of the effective mass extension coefficient of the iodine 
medium, the conversion factor of the image intensifier, the transfer function of the television system, the hardness of the incident radiation, and the electrical gain of the densitometer and recording system. In assessing relative flow, these latter factors could theoretically be maintained constantly and did not require separate calculations. Thus, the area under the videodilution curve was shown to be directly proportional to the amount of contrast material injected and inversely proportional to the flow at the injection site. With this type of analysis, coronary reactive hyperemia responses induced by contrast media were measured. Absolute flow was not calculated, and geometric measurements of vessel segments were not required. Accurate measurement of the delivered dose of contrast material necessitated slow injections to avoid reflux of contrast into the aortic root.

Several groups of investigators have applied modifications of the techniques mentioned above to the assessment of relative, regional coronary blood flow from digital subtraction coronary angiograms. ${ }^{200-203}$ Vogel et al have recently proposed a method of measuring reactive hyperemia based on digital radiographic enhancement of selective coronary arteriography ${ }^{200}$ (Fig 25).
Although previously determined from cineangiograms, the method is now performed on a rapid, real-time basis. ${ }^{204}$ The method utilizes ECGgated acquisition of a mask frame just prior to contrast injection and successive frames after injection which are obtained at the same period of the cardiac cycle to eliminate misregistration due to phasic cardiac motion. The progression of the contrast bolus through the arterial, myocardial, and venous phases are depicted by color coding of the transit relative only to the cardiac cycles. Cardiac cycle alterations caused by the contrast injections are prevented by atrial pacing. Large myocardial regions of interest are statistically analyzed to give the mean appearance time of contrast in the perfusion bed in absolute time units or cardiac cycle units. A second analysis is performed on a similarly acquired image after contrast or papaverineinduced hyperemic flow. ${ }^{205}$ In a normal situation, the mean appearance time is shorter if flow has increased. Shorter myocardial contrast appearance times are associated with higher flows so that the ratio of maximal to basal flow (ie, the reactive hyperemic ratio) is approximated by the myocardial appearance time measured during the control state, divided by the appearance time

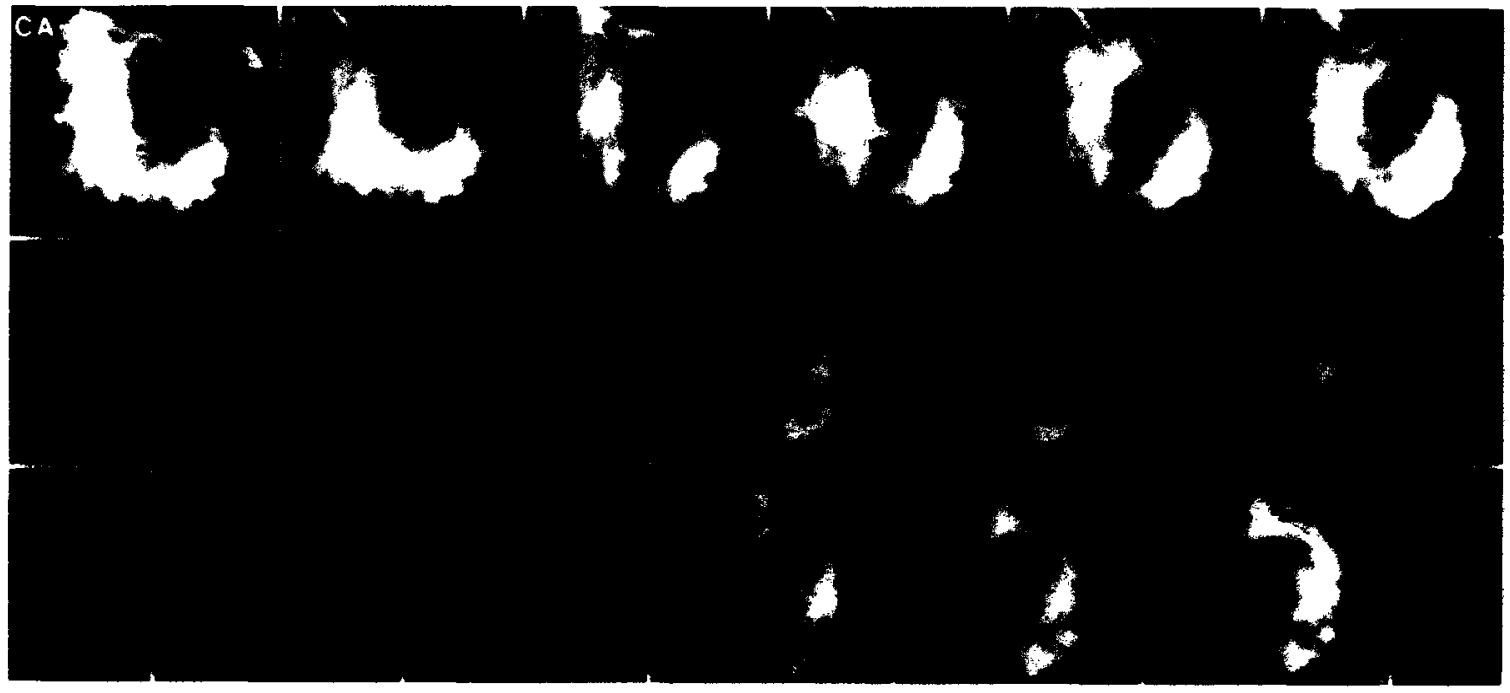

Fig 25. Digital enhancement of selective coronary arteriography obtained in the left anterior oblique projection from a patient with total occlusion of the left anterior descending coronary artery. Six consecutive pre-P wave end-diastolic cinearteriographic frames (top row, CA) are digitized into $256 \times 256$ eight-bit pixel matrices. The middle row shows the results of subtraction of pairs of consecutive frames (GID = gated interval difference), and the bottom row demonstrates mask-mode (MM) subtraction of the image series using the pre-contrast image (CA-O) as the mask. The temporal sequence of contrast transit is demonstrated and used to assess reletive coronary blood flow. (Reprinted with permission. ${ }^{200}$ ) CA, coronary artery: 0 , zero. 
during the hyperemic state. Initial validation demonstrated that directional changes in coronary sinus and great cardiac vein flow measured by the thermodilution technique during different levels of atrial pacing could be reflected by this technique $(r=.90)$, but with a systematic underestimation of the higher flow ratios. Recent animal studies ${ }^{204}$ have shown a similarly high correlation between electromagnetic flowmeter measurements of contrast-induced reactive hyperemia, and measurements obtained from simultaneous contrast appearance pictures, but high flow ratios were underestimated. Although further studies will be required to address these issues, the technique has been shown to be of potential clinical utility in assessing the adequacy of bypass grafts, ${ }^{205}$ coronary angioplasties, ${ }^{206,207}$ as well as in describing coronary steal phenomena ${ }^{208}$ and the effects of coronary collaterals on coronary dynamics. ${ }^{209}$ Furthermore, LeGrand et al ${ }^{210}$ have demonstrated that detection of impaired coronary reserve by this digital technique correlates well with evocation of regional wall motion or thallium perfusion defects during exercise. Moreover, the coronary flow reserve measurements showed greater correspondence with the stress test results than did the anatomical severity of coronary lesions. It should be stressed that this technique rests on the premises that regional blood flow is proportional to regional distribution volume divided by myocardial contrast appearance time, and that distributional volume, which is not mcasured, is presumed to remain constant. Under these conditions, flow is primarily related inversely to appearance time and, thus, the measurement of relative flows can theoretically be approximated by measured alterations in only this parameter under differing conditions of flow. Several investigations suggest that distributional volume is not constant after contrast injection and during pharmacologic vasodilation and, therefore, inaccuracies may result. ${ }^{25,211-219}$ Furthermore, the precision with which appearance time can be measured is limited by the temporal resolution of the technique which is based on cardiac cycle units. Consideration of these factors is currently under investigation to improve the accuracy of the method for measuring relative coronary flow. A recent study by Hodgson et al $^{219 a}$ demonstrates in dogs that by incorporating both the temporal and density changes occurring during contrast injection, flow reserve ratios can be measured that do not severely underestimate ratios obtained by electromagnetic flowmeter.

Other groups have proposed alternative analyses of digital coronary angiograms to estimate coronary flow dynamics. Whiting et $\mathrm{al}^{220}$ describe a method of integrated mask-mode subtraction followed by phasic subtraction to depict the transit of contrast material through epicardial and myocardial phases. Unlike the method described above, these invesigators determine the time course of pixel intensity over multiple small regions of the heart with 30 frame per second image acquisitions. Intensity information is corrected for background and to some extent, for the effects of scatter and veiling glare. This group and others have concentrated on analysis of the washout phase of contrast transit in determining relative, regional flow. ${ }^{201,202,203,221,222}$ Kruger et al measured blood flow velocity by measuring transit parameters obtained from temporal filtration processing of digital images, a technique which may also be suitable for measurement of coronary blood flow. ${ }^{223}$

Although these methods are attractive, it is important to realize that the use of contrast material as the indicator is problematic because of alterations of coronary blood flow during both the very early and later phases after contrast injection. ${ }^{194}$ Thus, both wash-in and wash-out methods are potentially invalidated if these effects are not considered. The technique proposed by Spiller et al ${ }^{195}$ is not as affected by this because analysis is performed in the very early postinjection phase (within $650 \mathrm{~ms}$ ) when flow alterations are minimal. Measurement of relative coronary flow is theoretically less technically demanding. But although this information is of physiologic significance, alterations in the flow ratios cannot be specifically attributed to alterations in either basal or hyperemic flow, except under controlled conditions. Thus, absolute flows would be preferable because altered flow ratios might be of widely disparate clinical signifcance. Furthermore, the techniques cannot currently provide information about the transmural distribution of flow.

Bursch et al $1^{224,225}$ also emphasize other potential problems in measuring flows by these techniques. For example, they required geometrical 
corrections of pin cushion distortion for measurements of relative flow. In addition, scatter was difficult to account for because of wide spatial variability in this parameter, as well as the temporal variability in scatter due to introduction of contrast material into the image field. The complexities of this issue have been addressed by other investigators as well. ${ }^{109-113,188}$ Despite these limitations, the methods represent promising attempts to extract more than just anatomical information from coronary angiograms. This potential ability to determine the functional significance of coronary lesions at rest is of great importance, particularly if it can be done rapidly and accurately, at the time of a single cardiac catheterization. The information is of both diagnostic and prognostic importance and may be expected to decrease the need for other ancillary tests (such as radionuclide exercise tests) that are frequently relied upon when the functional significance of a coronary lesion detected at angiography is questioned. The methods also show promise in defining, more physiologically, the adequacy of coronary angioplasty or bypass grafting.

\section{CONCLUSIONS}

Digital subtraction angiography is a new and growing field that has demonstrated significant promise in general medical and cardiac diagnosis. Further refinements in the hardware and software of digital imaging systems and advances in archival systems are required before filmbased imaging can be realistically replaced. This replacement will also be contingent upon the cost effectiveness of such systems. Although digital imaging lends itself readily to rapid and auto- mated quantitation, as well as to image manipulation methods that reduce contrast and/or radiation dose requirements and minimize the deleterious effects of patient motion on image quality, these attractions by themselves cannot currently justify full acceptance of digital angiography into cardiac practice. These benefits must be bolstered by a greater ease of implementation and an ability to extract new or more comprehensive information of potential benefit in patient management. In this regard, the ability to assess the physiologic significance of individual coronary stenosis is perhaps the most significant potential contribution of cardiac digjtal angiography to clinical cardiac practice. This will be achieved either through direct investigation of coronary flow relationships or by detection of subtle abnormalities of contractile function during stress. The benefit of more routine and automated assessment of pressure-volumethickness relationships in determining or assessing therapy remains largely unexplored. It seems apparent that these goals will most likely be achieved through the intra-arterial and not the IV applications of digital angiography. These capabilities would markedly amplify the investigative and diagnostic capabilities of cardiac catheterization laboratories while maintaining elficiency and a greater degree of safety, and while also helping to overcome the current inability of standard cardiac angiography to conveniently assess physiological parameters in relation to anatomy.

\section{ACKNOWLEDGMENT}

The authors wish to thank Diane Bauer for her expert secretarial assistance.

\section{REFERENCES}

I. Robb GP. Steinberg I: Visualization of the chambers of the heart, pulmonary circulation. and great blood vessels in man. AJR 1:1 17, 1938

2. Heintzen PH, Brennecke R, Bursch JH: Automated videoangiographic image analysis. Comput Cardiol (IEEE) $55-64,1975$

3. Brennecke R, Brown TK, Bursch JH: Digital processing of angiocardiographic image series using a minicomputer. Comput Cardiol (IEEE) 255-260, 1976

4. Frost MM, Fisher HD, Nudelman S, et al: A digital video acquisition system for extraction of subvisual information in diagnostic medical imaging. Proc SPIE, Appl Opt Instrument Med VI, Sept 25-27, Bellingham, WA 127:208$2.15,1977$
5. Kruger RA, Mistretta CA, Houk TL, et al: Computerized fluoroscopy in real time for noninvasive visualization of the cardiovascular system. Radiology 130:49-57, 1979

6. Kruger RA, Mistretta CA, Houk TL, et al: Computerized fluoroscopy techniques for intravenous study of cardiac chamber dynamics. Invest Radiol 14:279-287, 1979

7. Ovilt T, Christenson PC, Fisher HD, et al: Intravenous angiography using digital video subtraction: $\mathrm{X}$-ray imaging system. AJR 135:1141-1144, 1980

8. Crummy AB, Strother CM, Sackett JFf, et al: Computerized fluoroscopy, digital subtraction for intravenous angiocardiography and arteriography. AJR 135:1131-1140. 1980

9. Meaney TF, Weinstein MA, Buonocore E, et al: Digital 
subtraction angiography of the human cardiovascular system. AJR 1153-1160, 1980

10. Wood EH, Sturm RE, Sanders JJ: Data processing in cardiovascular physiology with particular reference to roentgen videodensitometry. Mayo Clin Proc 39:849-865, 1964

11. Nudelman S: Photoelectronic-digital radiology. Past, present and future, in Heintzen PH, Brennecke R (eds): Digital Imaging in Cardiovascular Radiology. Stuttgart/ New York, George Thieme Verlag, 1983, pp 1-14

12. Kruger RA: Introduction to digital radiographic imaging concepts, in Heintzen PH, Brennecke $\mathrm{R}$ (eds): Digital Imaging in Cardiovascular Radiology. Stuttgart/ New York, George Thieme Verlag. 1983, pp 14-23

13. Kruger RA: Basic physics of computerized fluoroscopy difference imaging, in Mistretta $C A$, Crummy $A B$, Strother CM, Sackett JF (eds): Digital subtraction arteriography: An application of computerized fluoroscopy. Chicago, Yearbook Medical Publishers, Inc, 1982, pp 16-22

14. Bailey NA, Crepeau RL: The capability of fluoroscopic systems to determine differential roentgen-ray absorption. Radiology 11 5:439-445, 1975

15. Kruger RA, Mistretta CA, Lancaster J, et al: A digital video image processor for real-time $\mathrm{x}$-ray subtraction imaging. Opt Eng 17:652-657, 1978

16. Bailey NA: Video techniques for $x$-ray imaging and data extraction from roentgenographic and fluoroscopic presentations. Med Phys 7:472-491, 1980

17. Ovitt TW, Fisher HD: Ideal configuration for intravenous digital subtraction angiography machine. Cardiovasc Intervent Radiol 6:300-302, 1983

18. Fisher HD, Nudelman S, Ovitt TW, et al: Specifications and features for the next generation intravenous angiography systems at the University of Arizona. Ann Radiol 26:543-547, 1983

19. Higgins CB: Effects of contrast materials on left ventricular function. Invest Radiol 15:S220-S231, 1980 (suppl)

20. Bettmann MA: Angiographic contrast agents: Conventional and new media compared. AJR 139:787-794, 1982

21. Fischer HW, Thomson KR: Contrast media in coronary angiography: A review. Invest Radiol 13:450-459, 1978

22. Rowe GG, I Iuston JH, Tuchman H, ct al: The physiologic effect of contrast media used for angiocardiography. Circulation 13:896-904, 1958

23. Read RC: Cause of death in cardioangiography. J Thorac Cardiovasc Surg 38:685-695, 1959

24. Brown R, Rahimtoola SH, Davis GD, et al: The effect of angiocardiographic contrast medium on circulatory dynamics in man. Circulation 33:234-240, 1965

25. Friesinger GC, Schaffer J, Criley JM, et al: Hemodynamic consequences of the injection of radiopaque material. Circulation 31:730-740, 1965

26. Rahimtoola SH, Duffy JP, Swan HJC: Hemodynamic changes associated with injection of angiocardiographic contrast medium in assessment of valvular lesions. Circulation 33:52-57, 1966

27. Fischer HW: Hemodynamic reactions to angiographic media. Radiology 91:66-73, 1968

28. Krovetz LJ, Mitchell BM, Neumaster T: Hemody- namic effects of rapidly injected hypertonic solutions into the heart and great vessels. Am Heart J 74:453-462, 1967

29. Gootman N, Rudolph AM, Buckley NM: Effects of angiographic contrast media on cardiac function. Am J Cardiol 25:59-65, 1970

30. Mullins CB, Leshin SJ, Mierzwiak DS, et al: Changes in left ventricular function produced by the injection of contrast media. Am Heart J 83:373-381, 1972

31. Hammermeister KE, Warbasse JR: Immediate hemodynamic effects of cardiac angiography in man. Am J Cardiol 31:307-314, 1973

32. Popia KA, Ross AM, Oravec JM, et al: Identification and description of separate mechanisms for two components of renografin cardiotoxieity. Circulation 58:520-528, 1978

33. Davis K, Kennedy JW, Kemp HG, et al: Complications of coronary arteriography from the collaborative study of coronary artery surgery (CASS). Circulation 59:11051112,1979

34. Ansell G, Tweedie McK, West CR, et al: The current status of reactions to intravenous contrast media. Invest Radiol 15:S32-S39, 1980 (suppl)

35. Mancini GBJ, Ostrander DR, Slutsky RA, et al: Intravenous vs. left ventricular injection of ionic contrast material: Hemodynamic implications for digital subtraction angiography. AJR 140:425-430, 1983

36. Sackett JF, Mann FA: Contrast media for digital subtraction arteriography of cerebral arteries, in Mistretta CA, Crummy AB, Strother CM, Sackett JF (eds): Digital Subtraction Arteriography: An Application of Computerized Fluoroscopy. Chicago, Yearbook Medical Publishers, Inc, 1982, pp 23-28

37. Seyferth W, Dilbat G, Zeitler E: Efficacy and safety of digital subtraction angiography with special reference to contrast agents. Cardiovasc Intervent Radiol 6:265-270, 1983

38. Almen T, Aspelin P: Cardiovascular effects of ionic monomeric, ionic dimeric and non-ionic contrast media: Effects in animals on myocardial contractial force, pulmonary and aortic blood pressure and aortic endothelium. Invest Radiol 10:557-563, 1975

39. DiDonato $M$, Cucchini BF, Baldi G, et al: Cardiovascular effects induced by the injection of a new nonionic contrast medium (iopamidol): Experimental study in dogs. Invest Radiol 14:309-315, 1979

40. Gerber KH, Higgins CB, Yuk Y-S, et al: Regional myocardial hemodynamic and metabolic effects of ionic and nonionic contrast media in normal and ischemic states. Circulation 65:1307-1314, 1982

41. Hirschfeld JW, Laskey W, Martin JL, et al: Hemodynamic changes induced by cardiac angiography with ioxaglate: Comparison with diatrizoate. JACC 2:954 957, 1983

42. Higgins CB, Gerber KH, Mattrey RA, et al: Evaluation of the hemodynamic effects of intravenous administration of ionic and nonionic contrast materials. Radiology 142:681-686, 1982

43. Mancini GB.I, Bloomquist JN, Bhargava V, et al: Hemodynamic and electrocardiographic effects in man of a new nonionic contrast agent (iohexol): Advantages over standard ionic agent. Am J Cardiol 51:1218-1222, 1983

44. Mancini GBJ, Atwood JE, Bhargava V, et al: Comparative effects of ionic and nonionic contrast materials on 
indexes of isovolumic contraction and relaxation in humans. Am J Cardiol 53:228-233, 1984

45. Sasayama S, Nonogi $H$, Kawai $C$, et al: Automated method for left ventricular volume measurement by cineventriculography with minimal doses of contrast medium. AnI J Cardiol 48:746-753, 1981

46. Kronenberg MW, Price RR, Smith CW, et al: Evaluation of left ventricular performance using digital subtraction angiography. Am J Cardiol 51:837-842, 1983

47. Nichols AB, Martin EC. Fles TP, et al; Validation of the angiographic accuracy of digital left ventriculography. Am J Cardiol 51:224-230, 1983

48. Tobis JM, Nalcioglu O, Johnston WD, et al: Correlation of 10-milliliter digital subtraction ventriculograms compared with standard cineangiograms. Am Heart J 105:946952,1983

49. Mistretta CA, Crummy AB, Strother CM: Digital angiography: A perspective. Radiology 139:272-276, 1981

50. Saddekni S, Sos TA. Sniderman KW, et al: Optimal injection technique for intravenous digital subtraction angiography. Radiology 150:655-659, 1984

51. Eskridge JM, Bccker GJ, Rabc FE, ct al: Digital vascular imaging: Practical aspects. Radiology 148:703-705, 1983

52. Modic MT, Weinstein MA, Pavlicek W, et al: Intravenous digital subtraction angiography: Peripheral versus central injection of contrast material. Radiolugy 147:71 I-715, 1983

53. Pond GD, Goldman S, Chernin MM, et al: The advantages of right atrial injections for intravenous digital subtraction angiography. Ann Radiol (Paris) 26:533-536, 1983

54. Burbank FH: Determinants of contrast enhancement for intravenous digital subtraction angiography. Invest Radiol 18:308-316, 1983

55. Brennecke R, Hahne HJ, Bursch JH, et al: Optimizaton of generalized subtraction operations. in Heintzen PH Brennecke R (eds): Digital Imaging in Cardiovascular Radiology. Stuttgart/New York, George Thieme Verlag, 1983 , pp 67-80

56. Hetzel PS, Swan HJC, Wood EH: Influence of injection site on arterial dilution curves of T-1824. J Appl Physiol 7:66-72, 1954-1955

57. Rubin DL, Burbank FH, Bradlcy BR, et al: An experimental evaluation of central vs. peripheral injection for intravenous digital subtraction angiography (IV-DSA). Invest Radiol 19:30-35, 1984

58. Riederer SJ, Kruger RA: Intravenous digital subtraction: A summary of recent developments. Radiology 147:633-638, 1983

59. Brody WR: Hybrid subtraction for improved arteriography. Radiology 141:828-831, 198

60. Higgins CB, Norris SL,Gerber KL, et al: Quantitation of left ventricular dimensions and function by digital video subtraction angiography. Radiology 144:461-469, 1983

61. Brennecke R, Bursch JH, Bogren HG, et al: Digital intravenous imaging techniques in pediatric cardiology, in Mistretta CA, Crummy AB, Strother CM, Sackett JF (eds): Digital Subtraction Arteriography: An Application of Computerized Fluoroscopy, Chicago, Year Book Medical Publishers, Inc, 1982, pp 133-141
62. Enzmann DR, Djang WT. Riederer SJ, et al: Lowdose, high-frame-rate versus regular-dose. low-frame-rate digital subtraction angiography. Radiology 146:669-676, 1983

63. Enzmann DR, Reiderer SJ, Hall AL, et al: Integration methods in post-process image manipulation in digital fluoroscopy. Ann Radiol (Paris) 26:557-563, 1983

64. Riederer SJ, Enzmann DR, Hall AL, et al: The application of matched filtering to $x$-ray exposure reduction in digital subtraction angiography: Clinical results. Radiology 146:349-354, 1983

65. Riederer S.J, Hall AI., Maier JK, et al: The technical characteristics of matched filtering in digital subtraction angiography. Med Phys 10:209-217, 1983

66. Riederer SJ, Brody WR, Enzmann DR, et al: Work in progress: The application of temporal filtering techniques to hybrid subtraction in digital subtraction angiography. Radiology 147:859-862, 1983

67. Gould RG. Lipton MJ, Mengers $P$, et al: Investigaltions of a video frame averaging digital subtraction fluoroscopic system. Proc Soc Photo-optical Instrumentation Engineers 314:184-191, 1981

68. Kruger RA: A method for time domain filtering using computerized fluoroscopy. Med Phys 8:466-469, 198 I

69. Kruger RA, Miller FJ, Nelson JA, et al: Digital subtraction angiography using a temporal bandpass filter: Associated patient motion properties. Radiology 145:315. 320,1982

70. Netson JA, Miller FJ, Kruger RA, et al: Digital subtraction angiography using a temporal bandpass filter: Initial clinical results. Radiology 145:309-313, 1982

7I. Nelson JA. Miller FJ, Mineau DE, et al: Clincal applications of digital filtration techniques, in Heintzgen PH, Brennecke R (eds): Digital Imaging in Cardiovascular $\mathbf{R}$ adiology. New York/Stuttgart. George Thieme Verlag, 1983, pp 183-192

72. Hardin CW, Kruger RA. Anderson FL, et al: Realtime digital angiocardiography using a temporal high-pass filter. Radiology 151:517-520, 1984

73. Bray BE, Hardin CW, Anderson FL, et al: Comparison of coronary anatomy by digital filtration and cineangiography methods. Circulation 68:40, 1983 (suppl) (abstr)

74. Guthaner DF. Brody WR, Lewis BD, et al: Clinical application of hybrid subtraction digital angiography: Preliminary results. Cardiovasc Intervent Radiol 6:290-294. 1983

75. Van Lysel MS, Dobbins JT, Peppler WW, et al: Work in progress: Hybrid temporal-energy subtraction in digital fluoroscopy. Radiology 147:869-874, 1983

76. Foley WD, Keyes GS. Smith DF, et al: Work in progress: Temporal energy hybrid subtraction in intravenous digital subtraction angiography. Radiology 148:265 271 . 1983

77. Mistretta CA, Peppler WW, Van Lysel M, et al: Recent advances in digital angiography. Ann Radiol (Paris) 26:537-542, 1983

78. Bogren HG, Bursch JH, Brennecke R, et al: Intravenous angiocardiography using digital image processing. 1 . Experience with axial projections in normal pigs. Invest Radiol 17:216-223, 1982

79. Kruger RA. Anderson RE, Koehier PR, ef at a 
method for the noninvasive evaluation of cardiovascular dynamics using digital radiographic device. Radiology 139:301-305, 1981

80. Vas R, Diamond GA, Levisman JA, et al: Computer enhanced digital angiography. Clin Cardiol 5:318-326, 1982

81. Bursch J, Heintzen PH, Simon R: Videodensitometric studies by a new method of quantitating the amount of contrast medium. Eur J Cardiol 1:437-446, 1974

82. Carey PH, Slutsky RA, Ashburn WL, et al: The validation of cardiac output by digital intravenous ventriculography in dogs: Correlation with thermodilution estimates. Radiology 143:623-626, 1982

83. Slutsky RA, Mancini GBJ, Norris S, et al: Digital intravenous ventriculography: Comparison of volumes form mask-mode and nonsubtracted images with thermodilution and sonocardiometric measurements. Invest Radiol 18:327334,1983

84. Radtke W, Bursch JH, Brennecke R, et al: Visualization of the left ventricular wall by digital angiocardiography. Eur Heart J 2:135-142, 1981

85. Radtke W, Bursch JH, Brennecke R, et al: Assessment of myocardial mass and infarction size by digital angiocardiography, in Heintzen $\mathrm{PH}$, Brennecke $\mathrm{R}$ (eds): Digital Imaging in Cardiovascular Radiology. Stuttgart/ New York, George Thieme Verlag, 1983, pp 233-240

86. Slutsky RA, Gerber KH, Higgins CB: Digital intravenous ventriculography: Analysis and validation of wall thickness changes during ischemia and inotropic stimulation in dogs. Am J Cardiol 50:874-880, 1983

87. Gerber KH, Slutsky RA, Ashburn WL, et al: Detection and assessment of severity of regional ischemic left ventricular dysfunction by digital fluoroscopy. Am Heart $\mathbf{J}$ $104: 27-35,1982$

88. Slutsky RA, Carey PH, Higgins CB: Effects of acute incremental volume overload on cardiac chamber size, function, and the pulmonary circulation: Analysis by digital intravenous angiography. Am Heart J 104:254-262, 1982

89. Vas R, Diamond GA, Forrester JS, et al: Computer enhancement of direct and venous-injected left ventricular contrast angiography. Am Heart J 102:719-728, 1981

90. Vas R, Diamond GA, Forrester JS, et al: Computerenhanced digital angiography: Correlation of clinical assessment of left ventricular ejection fraction and regional wall motion. Am Heart J 104:732-739, 1982

91. Tobis JM, Nalcioglu O, Johnston WD, et al: Left ventricular imaging with digital subtraction angiography using intravenous contrast injection and fluoroscopic exposure levels. Am Heart J 104:20-27, 1982

92. Goldberg HL, Borer JS, Moses JW, et al: Digital subtraction intravenous left ventricular angiography: Comparison with conventional intraventricular angiography. JACC 1:858-862, 1983

93. Norris SL, Slutsky RA, Mancini GBJ, et al: Comparison of digital intravenous ventriculography with direct left ventriculography for quantitation of left ventricular volumes and ejection fractions. Am J Cardiol 51:1399-1403, 1983

94. Felix R, Eichstadt H, Kempter H, et al: A comparison of conventional contrast ventriculography and digital subtraction ventriculography. Clin Cardiol 6:265-276, 1983

95. Nissen SE, Booth D, Waters $\mathbf{J}$, et al: Evaluation of left ventricular contractile pattern by intravenous digital subtrac- tion ventriculography: Comparison with cineangiography and assessment of interobserver variability. Am J Cardiol 52:1293-1298, 1983

96. Engels PHC, Ludwig JW, Verhoeven LAJ: Left ventricular evaluation by digital video subtraction angiography. Radiology 144:471-474, 1982

97. Bogren HG, Bursch JH, Brennecke R, et al: Choice of projection in intravenous digital angiocardiography, in Heintzen PH, Brennecke R (eds): Digital Imaging in Cardiovascular Radiology. Stuttgart/New York, George Thieme Verlag, 1983, pp 212-215, 1983

98. Fujita M, Sasayama S, Kwai C, et al: Automatic processing of cineventriculograms for analysis of regional myocardial function. Circulation 63:1065-1074, 1981

99. Seldin DW, Esser PD, Nichols AB, et al: Left ventricular volume determined from scintigraphy and digital angiography by a semi-automated geometric method. Radiology 149:809-813, 1983

99a. Mancini GBJ, Hodgson JMcB, Legrand V, et al: Quantitative assessment of global and regional left ventricular function with low-contrast dose digital subtraction ventriculography. Chest 87:598-602, 1985

100. Tobis J, Nalcioglu O, Johnston WD, et al: Digital angiography in assessment of ventricular function and wall motion during pacing in patients with coronary artery disease. Am J Cardiol 51:668-675, 1983

101. Mancini GBJ, Higgins CB: The evaluation of left ventricular function in ischemic heart disease by digital subtraction angiography, in Advances in Cardiac Imaging, Boston, G.K. Hall Medical Publishers, 1983, pp 113-133

102. Trenholm BG, Winter DA, Mymin D, et al: Computer determination of left ventricular volume using videodensitometry. Med Biol Eng Comput 10:163-173, 1972

103. Trenholm BG, Winer DA, Reimer GD, et al: Automated ventricular volume calculations from single plane images. Radiology 112:299-304, 1974

104. Tsakiris AG, Donald DE, Sturm RE, et al: Volume ejection fraction, and internal dimensions of left ventricle determined by biplane videometry. Fed Proc 28:1358-1367, 1969

105. Tobis J, Nalcioglu O, Seibert JA, et al: Measurement of left ventricular ejection fraction by videodensitometric analysis of digital subtraction angiograms. Am J Cardiol 52:871-875, 1983

106. Tobis J, Wong Y, Nalcioglu O, et al: Absolute left ventricular volume obtained with videodensitometry and digital subtraction angiography. JACC 1:616, 1983 (abstr)

107. Nissen S, Waters J, Booth D, et al: Determination of left ventricular ejection fraction by videodensitometric analysis of digital subtraction angiography: Experimental validation and correlation with area-length methods. JACC 1:617, 1983 (abstr)

108. Nissen S, Friedman B, Waters J, et al: Right ventricular ejection fraction by videodensitometry of intravenous digital subtraction angiograms: Experimental validation and initial clinical results. JACC 3:589, 1984 (abstr)

109. Shaw C-G, Ergun DL, Myerowitz PD, et al: A technique of scatter and glare correction for videodensitometric studies in digital subtraction videoangiography. Radiology 142:209-213, 1982

110. Kruger RA, Mistretta CA, Riederer SJ: Physical 
and technical considerations of computerized transmission fluoroscopy difference image. IEEE Trans Nucl Sci NS-28 1:205-212

111. Nalcioglu O, Seibert JA. Boone JM, et al: The effect of physical problems on the determination of ventricular ejection fraction by video densitometry, in Heintzen $\mathrm{PH}$, Brennecke R (eds): Digital Imaging in Cardiovascular Radiology, Stuttgart/New York, George Thieme Verlag, 1983, pp 104-115

112. Peppler WW, Van Lysel MS. Dobbins JT, et al: Progress report on the University of Wisconsin Digital Video Image Processor (DVIP 11), in: Heintzen PH, Brennecke R (eds): Digital Imaging in Cardiovascular Radiology, Stuttgart/New York, George Thieme Verlag, 1983, pp 56-66

113. Kruger RA: Image data acquisition, processing, storage and display. Cardiovasc Intervent Radiol 6:183-186, 1983

114. Chaitman BR, DeMoto H, Bristow JD, et al: Objective and subjective analysis of left ventricular angiograms. Circulation 52:420-425, 1975

115. Mancini GBJ, Norris SL, Peterson KL, et al: Quantitative assessment of segmental wall motion abnormalities at rest and after atrial pacing using digital intravenous ventriculography. JACC 2:70-76, 1983

116. Gelberg JH, Brundage BH, Glantz S, et al: Quantitative left ventricular wall motion analysis: $A$ comparison of area chord and radial methods. Circulation 59:991-1000. 1979

117. Karsch KR, Lamm U, Blanke H, et al: Comparison of nineteen quantitative methods for assessment of localized left ventricular wall motion abnormalities. Clin Cardiol $3: 123-128,1980$

118. Clayton PD, Jeppson GM, Klausner SC: Should a fixed external reference system be used to analyze left ventricular wall motion? Circulation 65:1518 1521, 1982

119. Sigel H, Nechwatal W, Kress $P$, et al: Interobserver and intermethod variation in evaluation of regional wall motion of the left ventricle. Cardiovase Intervent Radiol $6: 14-19,1983$

120. Widmann TF, Ashburn WL, Higgins CB, et al: Assessment of left ventricular wall motion by regional phase analysis of digital intravenous contrast fluoroangiography. Comput Cardiol (IEEE) 105-108, 1982

121. Norris SL, Widmann T, Mancini GBJ, et al: Assessment of wall motion by regional phase analysis of digital intravenous ventriculograms. Circulation 66:190, 1982 (suppl) (abstr)

122. Mancini GBJ, Higgins CB, Norris SL, et al: Cardiac imaging with digital subtraction angiography. Cardiovase Intervent Radiol 6:252-262, 1983

123. Mancini GBJ, Peterson KL, Gregoratos G, et al: Effects of atrial pacing on global and regional left ventricular function in coronary heart disease assessed by digital intravenous ventriculography. Am J Cardiol 53:456-461, 1984

124. Ross J Jr, Linhart JW, Braunwald E: Effects of changing heart rate in man by electrical stimulation of the right atrium: Studies at rest, during exercise and with isoproterenol. Circulation 32:549-558, 1965

125. Mahles $F$, Yoran $C$, Ross $\mathbf{J} \mathrm{J} r$ : Inotropic effect of tachycardia and post-stimulation potentiation in the conscious dog. Am J Physiol 22:569-575, 1974
126. Tomoike H, Franklin D, Ross IJr: Detection of myocardial ischemia by regional dysfunction during and after rapid pacing in conscious dogs. Circulation 58:48-56, 1978

127. Ricci DR, Orlick AE, Alderman EL, et al: Role of tachycardia as an inotropic stimulus in man. $J$ Clin Invest 63:695-703, 1979

128. Ricci DR, Orlick AE, Alderman EL, et al: Influence of heart rate on left ventricular ejection fraction in human beings. Am J Cardiol 44:447-451. 1979

129. Sasayama S. Takahashi $M$, Nakamura $M$, et al: Effects of diltiazem on pacing induced ischemia in conscious dogs with coronary stenosis: Improvement of post-pacing deterioration of ischemic myocardial function. Am J Cardiol 48:460-467, 1981

130. Johnson RA, Wasserman AG, Leibhoff RH, et al: Intravenous digital left ventriculography at rest and with atrial pacing as a screening procedure for coronary artery disease. JACC 2:905-910, 1983

131. Tobis JM, Nalcioglu O, Johnston WD, et al: Exercise digital subtraction angiograms in patients with coronary artery disease. Circulation 66:228, 1982 (suppl) (abstr)

132. Yiannikas J, Simpfendorfer C, Detrano R, et al: Stress digital subtraction angiography to assess presence of coronary artery disease in patients without myocardial infarction. Circulation 68:41, 1983 (suppi) (abstr)

133. Goldberg HL, Moses JW, Borer JS, et al: Exercise left ventriculography utilizing intravenous digital angiography. JACC 2:1092-1098, 1983

134. Spiller P, Deetjen W, Jehle J, et al: Quantitative evaluation of left ventricular function by digital subtractionand radionuclide angiocardiography at rest and during exercise. A comparison of both methods. Circulation 68:41, 1983 (suppl) (abstr)

135. Hugenholtz PG, Kaplan E, Hull E: Determination of left ventricular wall thickness by angiocardiography. Am Heart J 78:513-522, 1969

136. Eber LM, Greenberg HM, Cooke JM, et al: Dynamic changes in left ventricular free wall thickness in the human heart. Circulation 39:455-464, 1969

137. Rackley CE, Dodge HT, Cobel YD, et al: A method for determining left ventricular mass in man. Circulation 29:666-673, 1964

138. Dumesnil JG, Ritman EL. Frye RL, et al: Quantitative determination of left ventricular wall dynamics by roentgen videometry. Circulation 50:700-708, 1974

139. Dumesnil JG, Ritman EL, Davis GD, et at: Regional left ventricular wall dynamics before and after sublingual administration of nitroglycerin. $\Lambda \mathrm{m} \mathrm{J}$ Cardiol 36:419-425. 1975

140. Chesebro JH, Ritman EL, Frye RL, et al: Videometric analysis of regional left ventricular function before and after aortocoronary artery bypass surgery: Correlation of peak rate of myocardial wall thickening with late postoperative graft flows. J Clin Invest 58:1339-1347, 1976

141. St John Sutton MG, Tajik AJ, Smith HC, et al: Angina in idiopathic hypertrophic subaortic stenosis: A clinical correlate of regional left ventricular dysfunction: A videometric and echocardiographic study. Circulation $61: 561-568,1980$ 
142. Sandler H, Dodge HT: Left ventricular tension and stress in man. Circ Res 13:91-104, 1963

143. Peterson KL, Tsuji J, Johnson A, et al: Diastolic left ventricular pressure-volume and stress-strain relation in patients with valvular aortic stenosis and left ventricular hypertrophy. Circulation 58:77 -89, 1978

144. Carabello BA, Green I.H, Grossman W, et al: Hemodynamic determinants of prognosis of aortic valve replacement in critical aortic stenosis and advanced congestive heart failure. Circulation 62:42-48, 1980

145. Carabello BA, Nolan SP, Lockhart B: Assessment of preoperative left ventricular function in patients with mitral regurgitation: Value of the end-systolic wall stress to endsystolic volume ratio. Circulation 64:1212-1217, 1981

146. Engels PHC, Ludwig JW, Bruschke AVG, et al: Cardiac digital video subtraction angiography emphasizing left ventriculography, in Heintzen PH, Brennecke R (eds): Digital Imaging in Cardiovascular Radiology, New York/ Stuttgart, George Thieme Verlag, 1983, pp 192-204

147. Detre KM, Wright PH, Murphy ML, et al: Observer agreement in evaluating coronary angiograms. Circulation 52:979-986, 1975

148. Zir LM, Miller SW, Dinsmore RE, et al: Interobserver variability in coronary angiography. Circulation 52:627-632, 1976

149. DeRouen TA, Murray JA, Owen W: Variability in the analysis of coronary arteriograms. Circulation 55:324 328,1977

150. Sanmarco ME, Brooks SH, Blankenhorn $\mathrm{DH}$ : Reproducibility of a concensus panel in the interpretation of coronary angiograms. Am Heart J 96:430-437, 1978

151. Galbraith JE, Murphy ML, DeSoyza N: Coronary angiogram interpretation: Interobserver variability. JAMA 240:2053-2056, 1978

152. Fisher LK, Judkins MP, Lesperance J, et al: Reproducibility of coronary arteriographic reading in the coronary artery surgery study (CASS). Cathet Cardiovasc Diagn $8: 565-575,1982$

153. Vlodaver Z, Frech R, Van Tassel RA, ct al: Corrclation of the antemortem coronary arteriogram and the postmortem specimen. Circulation 47:162-169, 1973

154. Grondin CM, Dydra I, Pasternac A, et al: Discrepancies between cineangiographic and post-mortem findings in patients with coronary artery disease and recent myocardial revascularization. Circulation 49:703-708, 1974

155. Isner JM, Kishel J. Kent KM, et al: Accuracy of angiographic determination of left main coronary arterial narrowing. Circulation 63:1056-1064, 1981

156. Collins SM, Skorton DJ, Harrison DG, et al: Quantitative computer based videodensitometry and the physiological significance of a coronary stenosis. Comput Cardiol (IEEE) 219-222, 1982

157. White CW, Wright CB, Doty DB, et al: Does visual interpretation of the coronary arteriogram predict the physiologic importance of a coronary stenosis? N Engl J Med 310:819-824, 1984

158. Harrison DG, White CW, Hiratzka LF, et al: The value of lesion cross-sectional area determined by quantitative coronary angiography in assessing the physiologic significance of proximal left anterior descending coronary arterial stenoses. Circulation 69:1111-1119, 1984
159. Peppler WW, Kudva B, Dobbins JT, et al: A digitally controlled beam attenuator. Proc Soc Photo-optical Instrumentation Engineers 347:106-111, 1982

160. van der Werf T, Heethaar RM, Meijer FL: Coronary digital subtraction using the concept of apparent cardiac arrest. JACC 1:690, 1983 (abstr)

161. Myerowitz PD, Turnipseed WD, Shaw C-G, et al: Computerized fluoroscopy: New technique for the noninvasive evaluation of the aorta. coronary artery bypass grafts, and left ventricular function. J Thorac Cardiovasc Surg 83:65-73, 1982

162. Mycrowitz PD, Turnipsecd WD, Swanson DK, et al: Digital subtraction angiography as a method for screening for coronary artery disease during peripheral vascular angiography. Surgery 92:1042-1048, 1982

163. Myerowitz PD: Digital subtraction angiography: Present and future uses in cardiovascular diagnosis. Clin Cardiol 5:623-629, 1982

164. Drury JK, Gray R, Diamond GA, et al: Computer enhanced digital angiography visualizes coronary bypass grafts without need for selective injection. Circulation 66:229, 1982 (suppl) (abstr)

165. Brundage BH, Lipton MJ, Herfkens RJ, et al: Detection of patent coronary bypass grafts by computer tomography: A preliminary report. Circulation 61:826-831, 1980

166. Moncada R, Salinas M, Churchill R, et al: Patency of saphenous aortocoronary-bypass grafts demonstrated by computed tomography. N Engl J Med 303:503-505, 1980

167. Goldberg HL, Moses JW, Borer JS, et al: The role of digital subtraction angiography in coronary and bypass graft arteriography. Circulation 66:229. 1983 (suppl) (abstr)

168. Ross AM, Johnson RA, Katz RJ, et al: Diagnosis of coronary disease by aortic digital subtraction angiography. Circulation 63:43, 1983 (suppl) (abstr)

169. MacAlpin RN, Abbasi AS, Grothman JH, et al: Human coronary artery size during life. Radiology 108:567576,1973

170. Feldman RL, Pepine CJ, Curry RC, et al: Case against routine use of glyceryl trinitrate before coronary arteriography. Br Heart J 40:992-998, 1978

171. Feldman RL, Pepine CJ, Curry RC, et al: Coronary arterial responses to graded doses of nitroglycerin. Am J Cardiol 43:91-97, 1979

172. Feldman RL, Pepine CJ, Curry RC, et al: Quantitative coronary arteriography using $105 \mathrm{~mm}$ photospot angiography and an optical magnifying device. Cathet Cardiovasc Diagn 5:195-201, 1979

173. Gensini GG, Kelly AE, Da Costa BCB, et al: Quantitative angiography: The measurement of coronary vasomobility in the intact animal and man. Chest 60:522-530, 1971

174. Rafflenbeul W, Smith LR, Rogers WJ, et al: Quantitative coronary arteriography: Coronary anatomy of patients with unstable angina pectoris reexamined 1 year after optimal medical therapy. Am J Cardiol 43:699-707, 1979

175. Brown BG, Bolson F, Frimer M, et al: Quantitative coronary arteriography: Estimation of dimension, hemodynamic resistance, and atheroma mass of coronary artery lesions using the arteriogram and digital computation. Circulation 55:329-337, 1977

176. Sanders WJ, Alderman EL, Harrison DC: Coronary 
artery quantitation using digital image processing techniques. Comput Cardiol (IEEE) 15-20, 1979

177. Feldman RL, Nichols WW, Pepin CJ, et al: Hemodynamic significance of the length of a coronary arterial narrowing. Am J Cardiol 41:865-871, 1978

178. Feldman RL, Nichols WW, Pepine CJ, et al: Hemodynamic effects of long and multiple coronary arterial narrowings. Chest 74:280-285, 1978

179. Smith DN, Colfer H, Brymer JF, et al: A semiautomatic computer technique for processing coronary angiograms. Comput Cardiol (IEEE) 325-328, 1981

180. Reiber HJC, Booman F, Tan HS, et al: A cardiac image analysis system: Objective quantitative processing of angiograms. Comput Cardiol (IEEE) 239-242, 1978

181. Booman F, Reiber JHC, Bcrgrands JJ, ct al: Quantitative analysis of coronary occlusions from coronary cineangiograms. Comput Cardiol (IEEE) 177-180, 1979

182. Spears JR. Sandor T, Als AV, et al: Computerized image analysis for quantitative measurement of vessel diameter from cincangiograms. Circulation 68:453-461, 1983

183. Spears JR, Sandor T, Baim DS, et al: The minimum error in estimating coronary luminal cross-sectional area from cineangiographic diameter measurements. Cathet Cardiovasc Diagn 9:119-128, 1983

184. Crawford DW, Brooks SII. Barndt R, et al: Measurement of atherosclerotic luminal irregularity and obstruction by radiographic densitometry. Invest Radiol 12:307313. 1977

185. Sandor T, Als AV. Paulin S: Cinedensitometric measurement of coronary arterial stenosis. Cathet Cardiovasc Diagn 5:229-245, 1979

186. Kishon $Y$, Yerushalmi S, Deutsch V, et al: Measurement of coronary arterial lumen by densitometric analysis of angiograms. Angiology 30:304-312, 1979

187. Hoornstra K, Hanselman JMH, Holland WPJ, et al: Videodensitometry for measuring blood vessel diameter. Acta Radiol [Diagn] (Stockh) 21:115-164, 1980

188. Kruger RA: Estimation of the diameter of an iodine concentration within blood vessels using digital radiography devices. Med Phys 8:652-658, 1981

189. Nichols AB, Gabrich CFO, Fenoglio JJ, et al: Quantification of relative coronary arterial stenosis by cinevideodensitometric analysis of coronary arteriograms. Circulation 69:512-522, 1984

190. Rutishauser W. Bussman W-D. Noseda G, et al: Blood flow measurement through single coronary arteries by roentgen densitometry. Part I. A comparison of flow measured by radiologic techniques applicable in the intact organism and by electromagnetic flowmeter. A.JR 109:12-20, 1970

191. Rutishauser W, Noseda G, Bussman W-D: Blood flow measurements through single coronary arteries by roentgen densitometry. Part II. Right coronary artery flow in conscious man. AJR 109:21-24, 1970

192. Smith HC, Frye RI, Donald DE, et al: Roentgen videodensitometric measurement of coronary blood flow. Determination from simultaneous indicator-dilution curves to selected sites in the coronary circulation and in coronary artery saphenous vein grafts. Mayo Clin Proc 46:800-806, 1971

193. Smith HC, Sturm RE. Wood EH: Videodensitometric system for measurement of vessel blood flow, particularly in the coronary arteries, in man. Am J Cardiol 32:144-150. 1973

194. Hodgson JMcB, Mancini GBJ, Vogel RA: Characterization of changes in coronary blood flow during the first six seconds after contrast injections. JACC 3:589, 1984 (abstr)

195. Spiller P, Schmiel FK, Politz B, et al: Measurement of systolic and diastolic flow rates in the coronary artery system by $x$-ray densitometry. Circulation 68:337--347, 1983

196. Gould KL, Lipscomb K. Hamilton GW: Physiological basis for assessing critical coronary stenosis: Instantaneous flow response and regional distribution during coronary hyperemia as measures of coronary flow reserve. Am I Cardiol 33:87-94, 1974

197. Gould KL. Lipscomb K: Effects of coronary stenuses un coronary flow reserve and resistance. Am $\mathrm{J}$ Cardiol $34: 48 \quad 55,1974$

198. Foerster J, Link DP, Lantz BMT, et al: Measurement of coronary reactive hyperemia during clinical angiugraphy by video dilution technique. Acta Radiol [Diagn] (Stockh) 22:209-216, 1981

199. Foerster J, Lantz BMT, Holcroft JW, et al: Angiographic measurement of coronary blood flow by video dilution technique. Acta Radiol [Diagn] (Stockh) 22:121-127. 1981

200. Vogel R. LeFree M. Bates E, et al: Application of digital techniques to selective coronary arteriography: Use of myocardial contrast appearance time to measure coronary flow reserve. Am Heart J 107:153-164, 1984

201. Tobis J, Nalcioglu O. Boone J, et al: Vidcodenstiometric assessment of myocardial perfusion using digital subtraction angiography (DSA). Circulation 66:230, 1982 (suppl) (abstr)

202. Vas R, Whiting JS, Forrester JS, et al: Characterizattion of regional flow distribution in normal and abnormal coronary circulation. JACC 1:706, 1983 (abstr)

203. Bursch JH. Hahne JH, Beyer C, et al: Myocardial perfusion imaging by digital angiography. Circulation 68:42, 1983 (suppl) (abstr)

204. Hodgson JMcB, Aueron FM. Mancini GBJ, et al: A rapid digital angiographic technique for determining relative coronary blood flow: Validation and limitations. JACC 3:588, 1984 (abstr)

205. Bates ER. Vogel RA. LeFree MT, et al: Digital coronary radiographic determination of the coronary flow reserve provided by nonstenotic saphenous vein bypass grafts. JACC 1:619. 1983 (abstr)

206. ONeill WW, Walton JA, Bates ER, et al: Criteria for successful coronary angioplasty as assessed by alterations in coronary vasodilatory reserve. JACC 3:1382-1390, 1984

207. Aueron F. Gruentzig A, Vogel R, et al: Effects of successful percutaneous transluminal coronary angioplasty (PTCA) on acute and chronic coronary flow reserve. Circuliation 68:31, 1983 (suppl) (abstr)

208. Bates E. Vogel R, LeFree M. et al: Demonstration of intra- and inter-coronary steal by digital coronary radiography. Circulation 68:42, 1983 (suppl) (abstr)

209. LeGrand V, Aueron FM, Bates ER, et al: Reversibility of coronary collaterals and alteration in regional coronary flow reserve following successful angioplasty. Am J Cardiol $54: 453-454,1984$

210. LeGrand V, Vogel R, Gross MD, et al: The relation- 
ship of coronary flow reserve to stress thallium-201 myocardial perfusion and radionuclide ventriculography. $J \mathrm{Nucl}$ Med 25:28, 1984 (abstr)

211. Read RC, Johnson JA, Vick JA, et al: Vascular effects of hypertonic solutions. Circ Res 8:538-548, 1960

212. Lehan PH, Harman MA, Oldewurtel HA: Myocardial water shifts induced by coronary arteriography. J Clin Invest 42:950, 1963 (abstr)

213. Corsini G, Puri PS, Duran PVM, et al: Effect of nicotine on capillary flow and terminal vascular capacity of the heart in normal dogs and in animals with restricted coronary circulation. J Pharmacol Exp Ther 163:353-361, 1968

214. Branemark P-I, Jacobsson B, Sorensen SE: Microvascular effects of topically applied contrast media. Acta Radiol [Diagn] (Stockh) 8:547-559, 1969

215. Howe BB, Winbury MM: Effect of pentrinitrol, nitroglycerin, and propranolol on small vessel blood content of the canine myocardium. J Pharmacol Exp Ther 187:465474,1973

216. Weiss HR, Winbury MM: Nitroglycerin and chromonar on small-vessel blood content of the ventricular walls. Am J Physiol 226:838-843, 1974 (suppl)

217. Crystal GJ, Downey HF, Bashour FA: Small vessel and total coronary blood volume during intracoronary adenosine. Am J Physiol 241:194-201, 1981

218. Gould KL, Kelley KO: Physiologic significance of coronary flow velocity and changing stenosis geometry during coronary vasodilation in awake dogs. Circ Res 50:695704,1982
219. Tillmanns H, Steinhausen M, Dart A, et al: New aspects of myocardial capillary recruitment during hypoxia and reactive hyperemia. Circulation 676:43, 1982 (suppl) (abstr)

219a. Hodgson JMcB, Legrand V, Bates ER, et al: Validation in dogs of a rapid digital angiographic technique to measure relative coronary blood flow during routine cardiac catheterization. Amer J Cardiol 55:188-193, 1985

220. Whiting JS, Nivatpumin TL, Pfaff M, et al: Assessing the coronary circulation by digital angiography; Bypass graft and myocardial perfusion imaging, in Heintzen PH, Brennecke R (eds): Digital Imaging in Cardiovasular Radiology. Stuttgart/New York, George Thieme Verlag, 1983, pp 205-211

221. Nivatpumin T, Vas R, Pfaff $M$, et al: Changes in myocardial perfusion due to reactive hyperemia measured by digital angiography. Circulation 68:42, 1983 (suppl) (abstr)

222. Drury JK, Whiting JS, Pfaff JM, et al: Validation of myocardial blood flow measurements using digital subtraction angiography. JACC 3:588, 1984 (abstr)

223. Kruger RA, Bateman W, Liu PY, et al: Blood flow determination using recursive processing: A digital radiographic method. Radiology 149:293-298, 1983

224. Bursch JH, Hahne JH, Brennecke R, et al: Assessment of arterial blood flow measurements by digital angiography. Radiology 141:39-47, 1981

225. Bursch $\mathrm{JH}$ : Use of digitized functional angiography to evaluate arterial blood flow, Cardiovasc Intervent Radiol 6:303-310, 1983 